\title{
DIGITALCOMMONS
}

@WAYNESTATE —

Wayne State University

$10-1-2011$

\section{Variational Analysis of Marginal Functions with Applications to Bilevel Programming}

Boris S. Mordukhovich

Wayne State University, boris@math.wayne.edu

Nguyen Mau Nam

The University of Texas-Pan American, Edinburg, TX, nguyenmn@utpa.edu

Hung M. Phan

Wayne State University, pmhung@wayne.edu

\section{Recommended Citation}

Mordukhovich, Boris S.; Nam, Nguyen Mau; and Phan, Hung M., "Variational Analysis of Marginal Functions with Applications to Bilevel Programming" (2011). Mathematics Research Reports. Paper 91.

http://digitalcommons.wayne.edu/math_reports/91

This Technical Report is brought to you for free and open access by the Mathematics at DigitalCommons@WayneState. It has been accepted for inclusion in Mathematics Research Reports by an authorized administrator of DigitalCommons@WayneState. 


\title{
VARIATIONAL ANALYSIS OF MARGINAL FUNCTIONS WITH APPLICATIONS TO BILEVEL PROGRAMMING
}

\author{
BORIS S. MORDUKHOVICH, NGUYEN MAU NAM \\ and HUNG M. PHAN
}

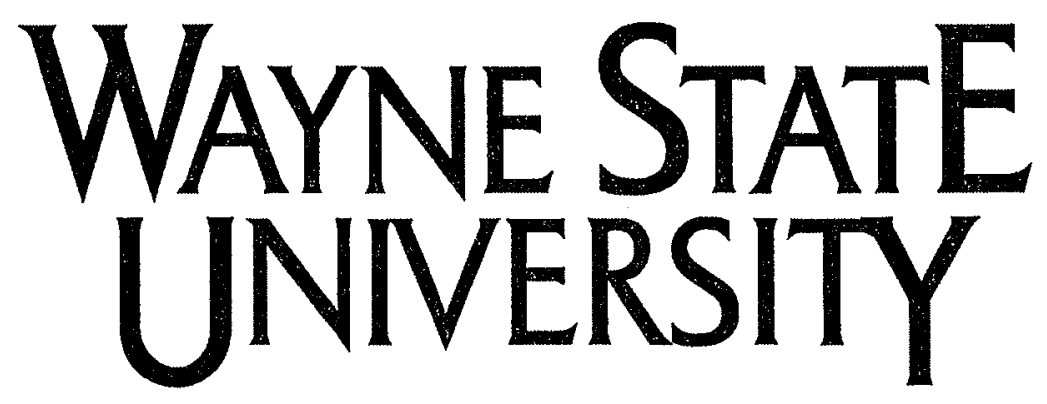

Detroit, MI 48202

Department of Mathematics

Research Report

\section{Series}

\#10 


\title{
VARIATIONAL ANALYSIS OF MARGINAL FUNCTIONS WITH APPLICATIONS TO BILEVEL PROGRAMMING
}

\author{
August 9, 2011 \\ BORIS S. MORDUKHOVICH ${ }^{1}$, NGUYEN MAU NAM ${ }^{2}$ and HUNG M. PHAN ${ }^{1}$.
}

\begin{abstract}
This paper pursues a twofold goal. First to derive new results on generalized differentiation in variational analysis focusing mainly on a broad class of intrinsically nondifferentiable marginal/value functions. Then the results established in this direction apply to deriving necessary optimality conditions for the optimistic version of bilevel programs that occupy a remarkable place in optimization theory and its various applications. We obtain new sets of optimality conditions in both smooth and smooth settings of finite-dimensional and infinite-dimensional spaces.

Keywords: Variational analysis and optimization, bilevel programming, generalized differentiation, marginal functions, optimality conditions
\end{abstract}

Mathematical Subject Classification 2000: Primary 49J52, 49J53; Secondary 90C30

\section{Introduction}

The major goal of this paper is to derive new necessary optimality conditions for a class of bilevel programs the importance of which has been well recognized in optimization theory and applications; see, e.g., the book by Dempe [6], the more recent publications $[7,8,10,26,28]$, and the bibliographies therein among other references that mostly concern bilevel programming in finite dimensions. Here we consider bilevel programs in infinite dimensions while all the results obtained are new in the standard framework of finite-dimensional spaces.

Recall that bilevel programming deals with problems of hierarchical optimization that consist of minimizing some upper-level cost/objective function $F: X \times Y \rightarrow \mathbb{R}$ defined on the product of Banach spaces $X$ and $Y$ subject to the upper-level constraints $x \in \Omega \subset X$ and some solution $y(x) \in Y$ of the parametric lower-level problem:

$$
\operatorname{minimize}_{y} \varphi(x, y) \text { subject to } y \in G(x)
$$

with the objective $\varphi: X \times Y \rightarrow \mathbb{R}$ and the constraint set-valued mapping $G: X \rightrightarrows Y$. In this paper we confine ourselves for simplicity to the case of lower-level inequality constraints

$$
G(x):=\{y \in Y \mid g(x, y) \leq 0\} \text { with } g=\left(g_{1}, \ldots, g_{p}\right): X \times Y \rightarrow \mathbb{R}^{p},
$$

defined on reflexive Banach spaces, where the vector inequality for $g$ is understood componentwise. The reader can observed from the proofs below that most of our results admit extensions to other types of constraints and more general Banach space settings.

Note that the bilevel problem formulated above is not fully determined if the solution map

$$
S(x):=\underset{y}{\operatorname{Argmin}}\{\varphi(x, y) \mid y \in G(x)\}, \quad x \in X,
$$

for the lower-level problem is set-valued, since in this case we did not specify how to choose a single-valued decision function $y(x)$. To deal with such a typical situation, the two major approaches have been designated in bilevel programming: optimistic and pessimistic.

\footnotetext{
${ }^{1}$ Department of Mathematics, Wayne State University, Detroit, MI 48202, USA (boris@math.wayne.edu, pmhung@wayne.edu). The research of these authors was partially supported by the US National Science Foundation under grant DMS-1007132, by the Australian Research Council under grant DP-12092508, and by the Portuguese Foundation of Science and Technologies under grant MAT/11109.

${ }^{2}$ Department of Mathematics, University of Texas-Pan American, TX 78539, USA (nguyenmn@utpa.edu). The research of this author was partially supported by the Simons Foundation under grant \#208785.
} 
The optimistic version of bilevel programs is formulated as follows:

$$
\text { minimize } F_{\text {opt }}(x) \text { subject to } x \in \Omega \text { with } F_{\text {opt }}(x):=\inf \{F(x, y) \mid y \in S(x)\} \text {, }
$$

which means that the decision $y(x)$ is chosen in $S(x)$ to benefit the objective $F_{\text {opt }}$. As usual, a point $\bar{x} \in \Omega$ is called a local optimistic solution/minimize of (1.4) if $F_{\text {opt }}(\bar{x}) \leq F_{\text {opt }}(x)$ for all $x \in \Omega$ sufficiently close to $\bar{x}$. Global optimistic solutions are defined in a similar way.

On the other hand, in some situation the decision $y(x)$ cannot be selected from the solution set $S(x)$ of the lower-level problem, and hence bad choices of $y(x)$ in $S(x)$ are not beneficial to the upper objective value. A natural approach in such cases is to reduce the damage caused by arbitrary choice of $y(x)$. This leads to formulating the pessimistic version of bilevel programs with replacing $F_{\text {opt }}$ in (1.4) by

$$
F_{p e s}(x):=\sup \{F(x, y) \mid y \in S(x)\}, \quad x \in X,
$$

where the pessimistic objective $F_{\text {pes }}$ represents the damage bound. Pessimistic local and global solutions are defined similarly to the optimistic case.

In this paper we concentrate on studying optimistic bilevel programs to derive new necessary optimality conditions for local optimistic solutions. Although many efforts have been made on this topic, it still remains a very attractive and challenging area of research. There are several approaches developed in the literature; see $[6,8,24,26,27]$ and the references therein. Let us briefly discuss the major ones to study local optimal solutions of problem (1.4).

The first idea is to replace the parametric lower-level problem (1.1)-(1.2) by the corresponding Karush-Kuhn-Tucker (KKT) system:

$$
\nabla_{y} \varphi(x, y)+\sum_{i=1}^{p} \lambda_{i} \nabla_{y} g_{i}(x, y)=0, g(x, y) \leq 0\langle\lambda, g(x, y)\rangle=0
$$

where $\lambda=\left(\lambda_{1}, \ldots, \lambda_{p}\right)$ is considered as a vector column. It is well known that the KKT system (1.5) provides necessary optimality conditions for the lower-level problem (1.1) under appropriate constraint qualifications. The resulting upper-level problem reduces to the so-called mathematical programs with equilibrium constraints (MPECs) of the type:

$$
\begin{aligned}
& \text { minimize } F(x, y) \text { subject to }(x, y, \lambda) \in \Omega \times Y \times \mathbb{R}^{p} \\
& \nabla_{y} \varphi(x, y)+\sum_{i=1}^{p} \lambda_{i} \nabla_{y} g_{i}(x, y)=0, \quad g(x, y) \leq 0, \quad\langle\lambda, g(x, y)\rangle=0 .
\end{aligned}
$$

Results in this direction under smoothness and convexity assumptions in the lower-level problem (1.1) can be found in [6] and the references therein. However, serious troubles arise in this approach when problem (1.1) is not convex since in this case the KKT system (1.5) may produce a point suspicious for local minima, which is not even feasible to the original problem. Another serious limitation in this approach is the violation of the classical Mangasarian-Fromovitz constraint qualification for the MPEC problem (1.6) considered as a standard nonlinear program.

A different approach to the study of optimistic bilevel programs, also known as Stackelberg games, was originally proposed by Outrata in [19] and then developed in [27] and other publications. The idea is to consider the optimal value function, also called the marginal function, of the lower-level problem (1.1) defined by

$$
\mu(x):=\inf _{y}\{\varphi(x, y) \mid y \in G(x)\} .
$$


Using this marginal function, the bilevel problem (1.4) can be reformulated as

$$
\begin{aligned}
& \text { minimize } F(x, y) \text { subject to } \\
& (x, y) \in \Omega \times Y, \quad g(x, y) \leq 0, \quad \varphi(x, y) \leq \mu(x) .
\end{aligned}
$$

Note that problem (1.8), which is of our main interest in this paper, is globally while not locally equivalent to the original optimistic formulation (1.4) in bilevel programming; see [6, 12] for more details. A detailed study of the original optimistic bilevel program (1.4) and the corresponding two-level value function is given [9].

The challenging issue in the value function approach is the nonsmoothness of problem (1.8) due to the intrinsic nondifferentiability of the value function (1.7), even when all the original data are smooth. Thus generalized differentiation plays a fundamental role in the study of bilevel programs via the value/marginal function approach. We refer the readers to $[14,15,16,18]$ and the bibliographies therein for various results on evaluating subdifferentials of marginal functions and their applications to optimization and sensitivity analysis. A crucial contribution to this approach was made by Ye and Zhu [27] who introduced the notion of partial calmness under which the bilevel program can be reduced to a problem of nondifferentiable programming with a penalized incorporating the troublesome constraint $\varphi(x, y) \leq \mu(x)$ into the new cost function. In this way necessary optimality conditions were established in [27], which were then extended and improved in $[8,10,11,13,24,25,26]$ and other publications. We particularly mention the recent study [10] that shows how the major reformulations of a bilevel program as a single-level optimization problem are effected by different constraint qualifications most of which fail in such reformulations, while the partial calmness occurs to be robust and important for applications to, e.g., demand adjustment problems in transportation. An interesting relaxation of partial calmness was developed in [13] with applications to a discretized obstacle control problem governed by partial differential equations of the elliptic type. Paper [28] proposed another approach to optimistic bilevel programs combining the aforementioned MPEC and value function ones and taking advantages of both for problems with smooth data. We also mention related developments in [11] for semi-infinite and infinite bilevel programs with DC (difference of convex) data and in $[1,26]$ for multiobjective bilevel programs.

Back to our study in this paper, we develop further the value function approach to optimistic bilevel programming following mainly the stream of [8], where necessary optimality conditions were obtained for (1.8) under partial calmness and the inner semicontinuity assumption on the solution map (1.3) to the lower-level problem. First we establish new results of generalized differential calculus and particularly for upper estimates of the convexified/Clarke subdifferential of a general class of marginal functions, not only those related to (1.8), which are of their independent interest for variational analysis and other applications. Employing the obtained marginal function results in the vein of [8] allows us derive new (in both finite and infinite dimensions) necessary optimality conditions for bilevel programs with smooth and also with Lipschitz continuous initial data. In particular, the obtained necessary optimality conditions for smooth bilevel programs do not require any convexification. We also present refined conditions ensuring the validity of partial calmness as well as several examples illustrating the strength of the new results. Finally, we explore advantages of lower and upper subgradients of the Hölder type for variational analysis of marginal functions with their applications to bilevel programming.

The rest of the paper is organized as follows. Section 2 presents basic definitions and preliminaries from generalized differentiation widely used in the sequel. In Section 3 we introduced and study new normal compactness conditions needed only in infinite dimensions and compare them with known conditions of this type. Section 4 is devoted to partial calmness in bilevel programming presenting new sufficient conditions for this property. In Section 5 we develop new calculus results for convexified subgradients by Clarke focusing on the derivation of refined upper 
estimates for this subdifferential of marginal functions that play an important role in what follows. Section 6 contains our major results on necessary conditions for bilevel programs with smooth and Lipschitzian data with no convexification. In the concluding Section 7 we develop calculus results for the general class of Hölder subgradients and incorporate them to deriving refined necessary optimality conditions for nonsmooth bilevel programs via limiting subgradients by Mordukhovich.

\section{Preliminaries from Generalized Differentiation}

In this section we overview, following the books $[14,15]$ with notation therein, some basic tools of generalized differentiation in variational analysis widely used in the paper. We also refer the reader to $[3,5,22,23]$ for related and additional material. Unless otherwise stated, all the spaces under consideration are Banach and reflexive with the generic notation $\|\cdot\|$ for their norms.

Given a set-valued mapping/multifunction $G: X \rightrightarrows X^{*}$ between a space $X$ and its topological dual $X^{*}$ with the canonical pairing $\langle\cdot, \cdot\rangle$, the construction

$$
\begin{aligned}
\underset{x \rightarrow \bar{x}}{\operatorname{Limsup}} G(x):=\left\{x^{*} \in X^{*} \mid\right. & \exists \text { sequences } x_{k} \rightarrow \bar{x} \text { and } x_{k}^{*} \stackrel{w}{\rightarrow} x^{*} \text { as } k \rightarrow \infty \\
& \text { with } \left.x_{k}^{*} \in G\left(x_{k}\right) \text { for all } k \in \mathbb{N}:=\{1,2, \ldots\}\right\} .
\end{aligned}
$$

signifies the sequential Painlevé-Kuratowski outer/upper limit of $F$ at $\bar{x}$, where $\stackrel{w}{\rightarrow}$ indicates the weak topology of the dual space $X^{*}$.

First we recall the major normal cone notions for an nonempty set $\Omega \subset X$. The regular/Fréchet normal cone to $\Omega$ at $\bar{x} \in \Omega$ is

$$
\widehat{N}(\bar{x} ; \Omega):=\left\{x^{*} \in X \mid \limsup _{x \rightarrow \bar{x}} \frac{\left\langle x^{*}, x-\bar{x}\right\rangle}{\|x-\bar{x}\|} \leq 0\right\},
$$

where $x \stackrel{\Omega}{\rightarrow} \bar{x}$ means that $x \rightarrow \bar{x}$ and $x \in \Omega$. We assume by convention that $\widehat{N}(x, \Omega):=\emptyset$ if $\bar{x} \notin \Omega$.

Note that $(2.2)$ may be trivial $(=\{0\})$ at boundary points of simple nonconvex sets in finite dimensions, and this construction does not possess satisfactory calculus rules. More robust normal cone notions to $\Omega$ at $\bar{x} \in \Omega$ are defined as follows: the limiting/Mordukhovich normal cone

$$
N(\bar{x} ; \Omega)=\operatorname{Limsup}_{x \rightarrow \bar{x}} \widehat{N}(x ; \Omega)
$$

via the limiting operation (2.1) and the convexified/Clarke normal cone

$$
\bar{N}(\bar{x} ; \Omega):=\operatorname{clco} N(\bar{x} ; \Omega)
$$

via the convex closure in $X^{*}$ of the collection of limiting normals (2.3). Note that for sets $\Omega$ locally closed around $\bar{x}$ the convexified construction (2.4) reduces in reflexive Banach spaces to the normal cone definition given in [5] due to [14, Theorem 3.57] and the classical Mazur theorem on weak closure of convex sets. We obviously have the relationships

$$
\widehat{N}(\bar{x} ; \Omega) \subset N(\bar{x} ; \Omega) \subset \bar{N}(\bar{x} ; \Omega),
$$

where both inclusions reduce to equalities for convex sets $\Omega$ while the three cones are essentially different in nonconvex settings. Note also that the cones $\widehat{N}(\bar{x} ; \Omega)$ and $\bar{N}(\bar{x} ; \Omega)$ are always convex but $N(\bar{x} ; \Omega)$ is not unless some regularity assumptions are imposed on $\Omega$. We say that $\Omega$ is normally regular at $\bar{x} \in \Omega$ if $\widehat{N}(\bar{x} ; \Omega)=N(\bar{x} ; \Omega)$. Since the set $N(\bar{x} ; \Omega)$ is convex and closed in this case, the latter implies that all the three cones in (2.5) agree with each other.

Considering further a set-valued mapping $G: X \rightrightarrows Y$ with the graph

$$
\operatorname{gph} G:=\{(x, y) \in X \times Y \mid y \in G(x)\},
$$


we use in the paper the two coderivative constructions for $G$ generated by the normal cones (2.3) and (2.4): the (normal) limiting coderivative of $G$ at $(\bar{x}, \bar{y}) \in \operatorname{gph} F$ and the convexified coderivative of $G$ at $(\bar{x}, \bar{y})$ defined by, respectively,

$$
\begin{gathered}
D^{*} G(\bar{x}, \bar{y})\left(y^{*}\right)=\left\{x^{*} \in X^{*} \mid\left(x^{*},-y^{*}\right) \in N((\bar{x}, \bar{y}) ; \operatorname{gph} G)\right\} \text { and } \\
\bar{D}^{*} G(\bar{x}, \bar{y})\left(y^{*}\right)=\left\{x^{*} \in X^{*} \mid\left(x^{*},-y^{*}\right) \in \bar{N}((\bar{x}, \bar{y}) ; \operatorname{gph} G)\right\} .
\end{gathered}
$$

If $G: X \rightarrow Y$ is single-valued and strictly differentiable at $\bar{x}$ (which is automatic when $G$ is smooth/continuously differentiable around this point), then we have

$$
D^{*} G(\bar{x})\left(y^{*}\right)=\bar{D}^{*} G(\bar{x})\left(y^{*}\right)=\left\{\nabla G(\bar{x})^{*} y^{*}\right\} \text { for all } y^{*} \in Y^{*}
$$

via the adjoint (strict) derivative linear operator $\nabla F(\bar{x})^{*}: Y^{*} \rightarrow X^{*}$, where $\bar{y}=G(\bar{x})$ is omitted in the coderivative notation for single-valued mappings.

Consider next an extended-real-valued function $\varphi: X \rightarrow \mathbb{R}:=\mathbb{R} \cup\{\infty\}$ with the domain $\operatorname{dom} \varphi:=\{x \in X \mid \varphi(x)<\infty\}$ and the associated epigraphical multifunction

$$
E_{\varphi}(x):=\{\alpha \in \mathbb{R} \mid \alpha \geq \varphi(x)\}
$$

for which we have $\operatorname{gph} E_{\varphi}=\operatorname{epi} \varphi:=\{(x, \alpha) \in X \times \mathbb{R} \mid \alpha \geq \varphi(x)\}$. Given $\vec{x} \in \operatorname{dom} \varphi$, the set

$$
\partial \varphi(\bar{x}):=D^{*} E_{\varphi}(\bar{x}, \varphi(\bar{x}))(1)=\left\{x^{*} \in X^{*} \mid\left(x^{*},-1\right) \in N((\bar{x}, \varphi(\bar{x})) ; \text { epi } \varphi)\right\}
$$

is known as the limiting/Mordukhovich subdifferential of $\varphi$ at $\bar{x}$ and the set

$$
\partial^{\infty} \varphi(\bar{x}):=D^{*} E_{\varphi}(\bar{x}, \varphi(\bar{x}))(0)=\left\{x^{*} \in X^{*} \mid\left(x^{*}, 0\right) \in N((\bar{x}, \varphi(\bar{x})) ; \text { epi } \varphi)\right\}
$$

as the corresponding singular subdifferential of $\varphi$ at this point. We refer the reader to $[14,22]$ the bibliographies therein for equivalent representations of constructions (2.9) and (2.10) and their various properties. Note that $\partial^{\infty} \varphi(\bar{x})=\{0\}$ if $\varphi$ is locally Lipschitzian around $\bar{x}$. Similarly we define the convexified/Clarke subdifferential (or the generalized gradient)

$$
\bar{\partial} \varphi(\bar{x}):=\bar{D}^{*} E_{\varphi}(\bar{x}, \varphi(\bar{x}))(1)=\left\{x^{*} \in X^{*} \mid\left(x^{*},-1\right) \in \bar{N}((\bar{x}, \varphi(\bar{x})) ; \operatorname{epi} \varphi)\right\}
$$

of $\varphi$ at $\bar{x}$ and the corresponding convexified singular subdifferential

$$
\bar{\partial}^{\infty} \varphi(\bar{x}):=\bar{D}^{*} E_{\varphi}(\bar{x}, \varphi(\bar{x}))(0)=\left\{x^{*} \in X^{*} \mid\left(x^{*}, 0\right) \in \bar{N}((\bar{x}, \varphi(\bar{x})) ; \text { epi } \varphi)\right\}
$$

of $\varphi$ at this point. In [5], the reader can find equivalent representations of (2.11) and its detailed study for local Lipschitzian functions. Note the following useful representation from [14, Theorem 3.57] of the convexified subdifferential (2.11) for lower semicontinuous (l.s.c.) functions via the subdifferential constructions (2.9) and (2.10):

$$
\bar{\partial} \varphi(\bar{x})=\operatorname{clco}\left[\partial \varphi(\bar{x})+\partial^{\infty} \varphi(\bar{x})\right]
$$

which reduces to $\bar{\partial} \varphi(\bar{x})=\operatorname{clco} \partial \varphi(\bar{x})$ when $\varphi$ is locally Lipschitzian around $\widetilde{x}$.

Finally in this section, we recall a property of set-valued mapping that plays a significant role in the results of this paper. A mapping $S: X \rightrightarrows Y$ is inner semicontinuous at $(\bar{x}, \bar{y}) \in \operatorname{gph} S$ if for every sequence $x_{k} \rightarrow \bar{x}$ with $S\left(x_{k}\right) \neq \emptyset$ there is a sequence $y_{k} \in S\left(x_{k}\right)$ such that $y_{k} \rightarrow \bar{y}$. For single-valued mappings this clearly reduces to the standard continuity of $S$ at $\bar{x}$. In the general case of set-valued mappings the above inner semicontinuity is automatic if $S$ is Lipschitzlike (or has the Aubin property) around $(\bar{x}, \bar{y})$; see [14, Theorem 4.10] and [22, Theorem 9.40] for coderivative characterizations of the latter property. We refer the reader to [8] for efficient conditions ensuring the validity of inner semicontinuity in the framework of bilevel programming. 


\section{Normal Compactness in Infinite Dimensions}

This section is devoted to the study of new "normal compactness" properties, which are certainly of their independent interest while are employed in what follows for calculus results and applications of the convexified generalized differential constructions reviewed in Section 2. These properties are automatic in finite-dimensional spaces; so the results of this section have meaning only in infinite dimensions.

Recall [14] that a set $\Omega \subset X$ is sequentially normally compact (or shortly SNC) at $\vec{x} \in \Omega$ if, for any sequences involved, we have the implication

$$
\left[x_{k} \stackrel{\Omega}{\rightarrow} \bar{x}, x_{k}^{*} \stackrel{w}{\rightarrow} 0, x_{k}^{*} \in \widehat{N}\left(x_{k} ; \Omega\right)\right] \Longrightarrow\left\|x_{k}^{*}\right\| \rightarrow 0 \text { as } k \rightarrow \infty .
$$

Accordingly, a set-valued mapping $G: X \rightrightarrows Y$ is $S N C$ at $(\bar{x}, \bar{y}) \in \operatorname{gph} G$ if its graph is SNC at this point, and an extended-real-valued function $\varphi: X \rightarrow \bar{R}$ with is sequentially normally epi-compact (SNEC) at $\bar{x} \in \operatorname{dom} \varphi$ if its epigraph is SNC at $(\bar{x}, \varphi(\bar{x}))$. These properties and their partial variants are comprehensively studied and applied in $[14,15]$.

For the purposes of this paper we need the following modifications of the above properties.

Definition 3.1 (SCNC). A set $\Omega \subset X$ is SEqUENTIALLY CONVEXLY NORMALLY COMPACT (SCNC) at $\bar{x} \in \Omega$ if we have the implication

$$
\left[x_{k} \stackrel{\Omega}{\rightarrow} \bar{x}, x_{k}^{*} \stackrel{w}{\rightarrow} 0, x_{k}^{*} \in \operatorname{co} N\left(x_{k} ; \Omega\right)\right] \Longrightarrow\left\|x_{k}^{*}\right\| \rightarrow 0 \text { as } k \rightarrow \infty
$$

for any sequences involved in (3.2). A mapping $G: X \rightrightarrows Y$ is SCNC at $(\bar{x}, \bar{y}) \in \operatorname{gph} G$ if its graph is $S C N C$ at this point. A function $\varphi: X \rightarrow \bar{R}$ is SEQUENTIALLY CONVEXLY EPI-COMPACT (SCNEC) at $\bar{x} \in \operatorname{dom} \varphi$ if its epigraph is $S C N C$ at $(\bar{x}, \varphi(\bar{x}))$.

It is obvious from (2.5) and the convexity of the cone $\widehat{N}(\bar{x} ; \Omega)$ that the SCNC property of a set implies the SNC one; the same holds of course for the corresponding properties for mappings and functions. Let us show that the opposite implication is violated in any Hilbert space.

Example 3.2 (SNC does not imply SCNC). Let $X$ be an arbitrary infinite-dimensional Hilbert space. Consider the set

$$
\Omega:=\{(x, y) \in X \times \mathbb{R} \mid y=-\|x\|\} .
$$

Since this set is the graph of a Lipschitz continuous function, it follows from [14, Corollary 1.69] that it is SNC at $(0,0)$. Now we demonstrate that (3.3) is not SCNC at this point. Indeed, we have by definition $(2.3)$ of the limiting normal cone $N((0,0) ; \Omega)$ and the structure of $(3.3)$ that

$$
\left\{\left(\frac{t x}{\|x\|}, t\right) \mid x \in X \backslash\{0\}, t \in \mathbb{R}\right\} \subset N((0,0) ; \Omega) .
$$

Observe that every pair $(x, \alpha) \in X \times \mathbb{R}$ can be represented as

$$
(x, \alpha)=\frac{1}{2}\left(\frac{t_{1} x}{\|x\|}, t_{1}\right)+\frac{1}{2}\left(-\frac{t_{2} x}{\|x\|}, t_{2}\right) \text { with } t_{1}:=\|x\|+\alpha \text { and } t_{2}:=\alpha-\|x\|,
$$

which $(x, \alpha) \in \operatorname{co} N((0,0), \Omega)$. Thus co $N((0,0), \Omega)=X \times \mathbb{R}$, which shows that the set $\Omega$ from (3.3) is not SCNC at $(0,0)$.

It is easy to check that the SCNC property holds at every point of a convex set with nonempty interior. Let us extend this result to a broad class of nonconvex sets. Given $\Omega \subset X$ with $\ddot{x} \in \Omega$, recall [20] that $v \in X$ is a hypertangent to $\Omega$ at $\bar{x}$ if for some $\delta>0$ we have

$$
x+t w \in \Omega \text { for all } x \in(\vec{x}+\delta \mathbb{B}) \cap \Omega, w \in v+\delta \mathbb{B} \text {, and } t \in(0, \delta) .
$$


Proposition 3.3 (SCNC property for sets with hypertangents). Assume that $\Omega \subset X$ admits a hypertangent at $\vec{x}$. Then $\Omega$ is $S C N C$ at this point.

Proof. Let $v$ be a hypertangent to $\Omega$ at $\bar{x}$, i.e., (3.4) holds with some number $\delta>0$. Fix $x \in(\bar{x}+\delta \mathbb{B}) \cap \Omega$ and $v^{*} \in \widehat{N}(x ; \Omega)$. The latter means that for any $\varepsilon>0$ there is $\eta>0$ such that

$$
\left\langle v^{*}, u-x\right\rangle \leq \varepsilon\|u-x\| \text { whenever } u \in \Omega \cap(x+\eta \mathbb{B}) .
$$

Using (3.4), for each $e \in \mathbb{B}$ and $t>0$ sufficiently small we have $x+t(v+\delta e) \in \Omega$. Hence

$$
t\left\langle v^{*}, v+\delta e\right\rangle \leq t \varepsilon\|v+\delta e\| \leq t \varepsilon(\|v\|+\delta),
$$

which ensures in turn the estimate

$$
\delta\left\langle v^{*}, e\right\rangle \leq \varepsilon(\|v\|+\delta)-\left\langle v^{*}, v\right\rangle \text { for all } e \in \mathbb{B},
$$

and thus $\delta \| v^{*}|| \leq \varepsilon(\|v\|+\delta)-\left\langle v^{*}, v\right\rangle$. Letting $\varepsilon \downarrow 0$ gives us the inequality

$$
\delta\left\|v^{*}\right\| \leq\left\langle v^{*},-v\right\rangle .
$$

Next we fix a limiting normal $x^{*} \in N(x ; \Omega)$ with some $x \in(\bar{x}+\gamma \mathbb{B}) \cap \Omega$ as $\gamma<\delta$. By definition (2.3) there are sequences $\left\{x_{k}\right\}_{k \in \mathbb{N}}$ in $(\bar{x}+\delta \mathbb{B}) \cap \Omega$ and $\left\{x_{k}^{*}\right\}_{k \in N}$ in $X^{*}$ such that $x_{k} \stackrel{\Omega}{\rightarrow} x$ and $x_{k}^{*} \stackrel{w}{\rightarrow} x^{*}$ with $x_{k}^{*} \in \widehat{N}\left(x_{k} ; \Omega\right)$ for every $k \in \mathbb{N}$. Employing (3.5) for $v^{*}=x_{k}^{*}$ and the lower semicontinuity of the norm function in the weak topology of $X$, we get

$$
\delta\left\|x^{*}\right\| \leq \delta \liminf _{k \rightarrow \infty}\left\|x_{k}^{*}\right\| \leq \liminf _{k \rightarrow \infty}\left\langle x_{k}^{*},-v\right\rangle=\left\langle x^{*},-v\right\rangle,
$$

which ensures the inclusion

$$
N(x ; \Omega) \subset\left\{x^{*} \in X^{*} \mid \delta\left\|x^{*}\right\| \leq\left\langle x^{*},-v\right\rangle\right\} \text { for every } x \in(\bar{x}+\gamma \mathbb{B}) \cap \Omega .
$$

The convexity of the set on the right-hand side of the latter inclusion implies that

$$
\operatorname{co} N(x ; \Omega) \subset\left\{x^{*} \mid \delta\left\|x^{*}\right\| \leq\left\langle x^{*},-v\right\rangle\right\} .
$$

Take now any sequences $x_{k} \stackrel{\Omega}{\rightarrow} \bar{x}$ and $x_{k}^{*} \stackrel{w}{\rightarrow} 0$ with $x_{k}^{*} \in \operatorname{co} N\left(x_{k} ; \Omega\right)$ for all $k \in \mathbb{N}$. Then inclusion (3.6) ensures that estimate (3.5) holds along this sequence $\left\{x_{k}^{*}\right\}_{k \in \mathbb{N}}$, which clearly yields that $\left\|x_{k}^{*}\right\| \rightarrow 0$ as $k \rightarrow \infty$ and thus justifies the SCNC property of $\Omega$ at $\bar{x}$.

Note that the existence of hypertangents at the reference point of a set is just a sufficient condition for the validity of the SCNC property of the set at this point. Indeed, it is well known that there are sets in finite dimensions, which do not have hypertangents at some of their points while all such sets are surely SCNC.

Finally in this section, we show that every local Lipschitzian function is SCNEC at the reference point. This result is widely used in what follows.

Proposition 3.4 (SCNEC property of local Lipschitzian functions). Let $\varphi: X \rightarrow \overline{\mathbb{R}}$ be locally Lipschitzian around $\vec{x} \in \operatorname{dom} \varphi$. Then it is $S C N E C$ at the point.

Proof. Consider any sequences $\left(x_{k}, \mu_{k}\right) \rightarrow(\bar{x}, \varphi(\bar{x}))$ and $\left(x_{k}^{*},-\lambda_{k}\right) \stackrel{w}{\rightarrow}(0,0)$ as $k \rightarrow \infty$ with $\left(x_{k}, \mu_{k}\right) \in \operatorname{epi} \varphi$ and $\left(x_{k}^{*},-\lambda_{k}\right) \in \operatorname{co} N\left(\left(x_{k}, \mu_{k}\right)\right.$; epi $\left.\varphi\right)$ for all $k \in \mathbb{N}$, where $\lambda_{k} \geq 0$ by [14, Proposition 1.76]. By the continuity of $\varphi$, suppose with no loss of generality that $\mu_{k}=\varphi\left(x_{k}\right)$ for 
all $k$. Then for each $k$ we find pairs $\left(u_{i k}^{*},-\nu_{i k}\right) \in N\left(\left(x_{k}, \varphi\left(x_{k}\right)\right)\right.$; epi $\left.\varphi\right)$ with $\nu_{i k} \geq 0$ and numbers $t_{i k} \geq 0$ as $i=1, \ldots, m=m(k) \in \mathbb{N}$ such that

$$
\sum_{i=1}^{m} t_{i k}=1 \text { and }\left(x_{k}^{*},-\lambda_{k}\right)=\sum_{i=1}^{m} t_{i k}\left(u_{k}^{*},-\nu_{i k}\right), \quad k \in \mathbb{N} .
$$

Since $\varphi$ is locally Lipschitzian around $\bar{x}$ with some constant $\ell>0$, it follows from [14, Corollary 1.81] that $\left\|u_{i k}^{*}\right\| \leq \ell \nu_{i k}$. This implies by (3.7) that

$$
\left\|x_{k}^{*}\right\| \leq \sum_{i=1}^{m} t_{i k}\left\|u_{i k}^{*}\right\| \leq \ell \sum_{i=1}^{m} t_{i k} \nu_{i k}=\ell \lambda_{k} \text { for all } k \in \mathbb{N} .
$$

Recall that $\lambda_{k} \downarrow 0$ by the choice of the sequences above, and thus we get from (3.8) that $\left\|x_{k}^{*}\right\| \rightarrow 0$ as $k \rightarrow \infty$, which justifies the SCNEC property of $\varphi$ at $\bar{x}$.

\section{Partial Calmness in Bilevel Programming}

As mentioned in Section 1, partial calmness plays a significant role in the study of optimistic bilevel programs. In this section we derive new sufficient conditions for this property, which complement those obtained in $[8,10,27]$.

Unless otherwise specified, we focus on the optimistic bilevel problem (1.4) in the form

$$
\begin{array}{ll}
\text { minimize } & F(x, y) \quad \text { subject to } x \in \Omega, y \in G(x), \\
& \text { and } \varphi(x, y)-\mu(x) \leq 0,
\end{array}
$$

where $\mu(x)$ is the marginal function (1.7), and where the constraint sets are defined by

$$
\begin{aligned}
G(x) & :=\left\{y \in Y \mid g_{i}(x, y) \leq 0, i=1, \ldots, p\right\}, \\
\Omega & :=\left\{x \in X \mid h_{j}(x) \leq 0, j=1, \ldots, m\right\} .
\end{aligned}
$$

The perturbed version of (4.1) parameterized by $u \in \mathbb{R}$ is written as

$$
\begin{array}{ll}
\text { minimize } & F(x, y) \\
& \text { subject to } x \in \Omega, y \in G(x), \\
& \text { and } \varphi(x, y)-\mu(x)+u=0 .
\end{array}
$$

Following [27], the unperturbed program (4.1) is said to be partially calm at its given feasible point $(\bar{x}, \bar{y})$ if there exist a constant $\lambda>0$ and a neighborhood $U$ of $(\bar{x}, \bar{y}, 0) \in X \times Y \times \mathbb{R}$ such that for all $(x, y, u) \in U$ feasible to (4.4) we have

$$
F(x, y)-F(\bar{x}, \bar{y})+\lambda|u| \geq 0 .
$$

Our interest on the partial calmness is drawn by its capacity to "move" the marginal function constraint $\varphi(x, y)-\mu(x)$ from the feasible set to the upper objective function by penalization. Thus the optimistic bilevel program reduces in this way to a single-level problem of nondifferentiable programming. This is ensured by the following assertion from [27].

Proposition 4.1 (penalization under partial calmness). Let $(\bar{x}, \bar{y})$ be a local optimal solution to problem (4.1). This problem is partially calm at $(\bar{x}, \bar{y})$ if and only if there exists $\kappa>0$ such that $(\bar{x}, \bar{y})$ is a local optimal solution of the partially penalized problem

$$
\begin{aligned}
& \operatorname{minimize} F(x, y)+\kappa(\varphi(x, y)-\mu(x)) \\
& \text { subject to } x \in \Omega, y \in G(x) .
\end{aligned}
$$


A well-recognized sufficient condition for partial calmness is the concept of weak sharp minima whose definition employed below is as follows: Given $\Omega \subset Y$, the set $S \subset \Omega$ is called a set of local weak sharp minima with respect to $\Omega$ for a function $\varphi: Y \rightarrow \mathbb{R}$ at $\bar{y} \in S$ with modulus $\alpha>0$ if

$$
\varphi(y) \geq \varphi(\bar{y})+\alpha \operatorname{dist}(y ; S) \text { for all } y \in \Omega \text { near } \bar{y} \text {. }
$$

We refer the reader to [4] and the bibliographies therein for more details on this notion and its applications to variational analysis and optimization and to the recent paper [29] for complete characterizations of local weak sharp minima via generalized differentiation with new applications to semi-infinite programming and complementarity. The next proposition presents the precise formulation needed in what follows and provides its simple proof.

Proposition 4.2 (partial calmness from local weak sharp minima). Let $(\bar{x}, \bar{y})$ be a local optimal solution to the bilevel program (4.1) such that

$$
\varphi(x, y)-\mu(x) \geq \alpha \operatorname{dist}(y ; S(x)) \text { with some } \alpha>0
$$

for all $(x, y)$ near $(\bar{x}, \bar{y}), x \in \Omega$, and $y \in G(x)$. Then problem (4.1) is partially calm at $(\bar{x}, \bar{y})$.

Proof. Picking any pair $(x, y)$ feasible to the perturbed problem (4.4) and sufficiently close to $(\bar{x}, \bar{y})$, we have $x \in \Omega, y \in G(x)$, and $\varphi(x, y)-\mu(x)+u=0$ with small $u \in \mathbb{R}$. By assumption (4.6), find $\tilde{y} \in S(x)$ such that

$$
\varphi(x, y)-\mu(x) \geq \frac{\alpha}{2}\|y-\tilde{y}\| \geq 0 .
$$

Since $(\bar{x}, \bar{y})$ is a solution to $(4.1)$, we have

$$
F(x, y)-F(\bar{x}, \bar{y}) \geq F(x, y)-F(x, \tilde{y}) \geq-\ell\|y-\tilde{y}\| \geq-\frac{2 \ell}{\alpha}(\varphi(x, y)-\mu(x))=-\lambda|u|,
$$

where $\lambda:=2 \ell / \alpha$ for the Lipschitz constant $\ell$ of $F$. This justifies the partial calmness.

In the next two lemmas we establish sufficient conditions for local weak sharp minima, which are of their own interest while implying the required partial calmness of the bilevel program (4.1) by virtue of Proposition 4.2.

Lemma 4.3 (sufficient conditions for local weak sharp minima, I). Let $f: \mathbb{R}^{m} \rightarrow \mathbb{R}$, and let $\bar{y}$ be a local optimal solution to the problem:

$$
\text { minimize } f(y) \text { subject to } y \in \Omega:=\left\{y \in \mathbb{R}^{m} \mid g_{i}(y) \leq 0, i=1, \ldots, p\right\} \text {. }
$$

Impose the following assumptions:

(i) The functions $f$ and $g_{i}$ as $i \in I(\bar{y}):=\left\{i \mid g_{i}(\bar{y})=0\right\}$ are Fréchet differentiable at $\bar{y}$.

(ii) The Karush-Kuhn-Tucker condition

$$
\nabla f(\bar{y})+\sum_{i \in I(\bar{y})} \lambda_{i} \nabla g_{i}(\bar{y})=0 \text { for some } \lambda_{i} \geq 0
$$

holds with $\cap_{i \in I} \operatorname{ker} \nabla g_{i}(\bar{y})=\{0\}$, where $I:=\left\{i \mid \lambda_{i}>0\right\}$.

Then there exists a positive constant $\alpha>0$ such that

$$
f(y)-f(\bar{y}) \geq \alpha\|y-\bar{y}\| \text { for all } y \in \Omega \text { near } \bar{y} .
$$

Consequently, the vector $\bar{y}$ is an isolated local minimizer for $f$ on $\Omega$, and the function $f$ admits a set of local weak sharp minima over $\Omega$. 
Proof. To justify the validity of (4.8) with some constant $\alpha>0$, suppose on the contrary that there exist $\bar{y} \neq y_{k} \in \Omega$ with $y_{k} \rightarrow \bar{y}$ as $k \rightarrow \infty$ such that

$$
f\left(y_{k}\right)-f(\bar{y}) \leq \frac{1}{k}\left\|y_{k}-\bar{y}\right\| \text { for all } k \in \mathbb{N} .
$$

Let $d_{k}:=\frac{y_{k}-\bar{y}}{\left\|y_{k}-\bar{y}\right\|}$ and without loss of generality assume that $d_{k} \rightarrow d$ as $k \rightarrow \infty$ with $\|d\|=1$. It follows from (4.9) by the Fréchet differentiability of $f$ at $\bar{y}$ that

$$
\langle\nabla f(\bar{y}), d\rangle \leq 0
$$

On the other hand, the Fréchet differentiability of the active constraint gradients in (i) yields

$$
\left\langle\nabla g_{i}(\bar{y}), d\right\rangle \leq 0 \text { for all } i \in I(\bar{y})
$$

Using the last two inequalities and the KKT condition in (ii), we get that

$$
0 \leq-\langle\nabla f(\bar{y}), d\rangle=\sum_{i \in I} \lambda_{i}\left\langle\nabla g_{i}(\bar{y}), d\right\rangle \leq 0
$$

which implies that $\left\langle\nabla g_{i}(\bar{y}), d\right\rangle=0$ for all $i \in I$ and hence $d=0$ by the kernel condition in (ii). This contradiction completes the proof of the lemma.

Observe that the kernel assumption in (ii) is essential for the validity of Lemma 4.3. Indeed, consider problem (4.7) with $f, g: \mathbb{R}^{2} \rightarrow \mathbb{R}$ defined by

$$
f\left(y_{1}, y_{2}\right):=y_{1}^{2}-y_{2} \text { and } g\left(y_{1}, y_{2}\right):=y_{2} \text {. }
$$

Then $\Omega=\mathbb{R} \times \mathbb{R}_{-}$, and the origin $\vec{y}:=(0,0)$ is the only solution to this problem. Since

$$
\operatorname{ker} \nabla g(\bar{y})=\mathbb{R} \times\{0\},
$$

the kernel assumption in (ii) of Lemma 4.3 is violated. It is easy to see that

$$
f(y)-f(\bar{y})=v^{2} \text { and }\|y-\bar{y}\|=v \text { for any } \mathrm{y} y=(v, 0) \in \Omega \text {. }
$$

Since $v>0$ can be chosen arbitrarily small, the conclusion in (4.8) does not hold.

The second lemma gives another set of conditions for local weak sharp minima in (4.1).

Lemma 4.4 (sufficient conditions for local weak sharp minima, II). Let $S$ be a set of optimal solutions to problem (4.7). Assume that the functions $f, g_{i}: \mathbb{R}^{m} \rightarrow \mathbb{R}$ are continuously differentiable around any point of $S$ and that there exist a set $D$ of unit directions and a number $\eta>0$ such that for any small $\varepsilon \geq 0$ we have:

(i) $\Omega \cap(S+\varepsilon \mathbb{B}) \subset S+$ cone $[D+o(\varepsilon) \mathbb{B B}]$ and

(ii) $\langle\nabla f(y), d\rangle>\eta$ whenever $d \in D$ and $y \in S+\varepsilon \mathbb{B}$, where we suppose by convention that $o(\varepsilon)=0$ if $\varepsilon=0$.

Then $S$ is a set of local weak sharp minima of the function $f$ on $\Omega$.

Proof. Picking any $y \in \Omega \cap(S+\varepsilon \mathbb{B})$ and letting $\varepsilon^{\prime}:=\operatorname{dist}(y ; S)$, we also have $y \in \Omega \cap\left(S+\varepsilon^{\prime} \mathbb{B}\right)$. Thus it can be assumed that $y \in \Omega \cap(S+\varepsilon \mathbb{B})$ and $\varepsilon=\operatorname{dist}(y ; S)$. It follows from (i) that there are $\bar{y} \in S, \lambda>0$, and $d \in D+o(\varepsilon) \mathbb{B}$ such that

$$
y=\bar{y}+\lambda d .
$$


This implies that $\lambda\|d\|=\|y-\bar{y}\| \geq \varepsilon$. Since $D$ contains only unit directions, we may suppose also that $\|d\|=1+o(\varepsilon)$. It follows that $\lambda \geq \varepsilon+o(\varepsilon)$. The smoothness of $f$ allows us to find $y^{\prime}$ lying on the segment of $\bar{y}$ and $y$ satisfying the relationships

$$
f(y)-f(\bar{y})=\nabla f\left(y^{\prime}\right) \lambda d \geq \frac{1}{2} \varepsilon \eta=\frac{\eta}{2} \operatorname{dist}(y ; S) .
$$

This ensures that $S$ is a set of local weak sharp minima of $f$ over $\Omega$.

Unifying the above discussions, we arrive at the following verifiable conditions ensuring partial calmness in bilevel programming.

Theorem 4.5 (sufficient conditions for partial calmness). Let $(\bar{x}, \bar{y})$ be a local optimal solution to the bilevel program (4.1), where all the functions involved are smooth around the reference points. Suppose further that there exists a bounded set $K \subset Y$ such that either one of the following assumptions holds for all $x$ around $\bar{x}$ :

(i) We have the inclusion $S(x) \subset K$ for the solution sets to the lower-level problem in (4.1), and condition (ii) in Lemma 4.3 is satisfied for all $y \in S(x)$.

(ii) All the assumptions of Lemma 4.4 are satisfied for the lower-level problem with $\eta>0$ and $o(\varepsilon)$ being independent of $x$.

Then the bilevel program (4.1) is partially calm at $(\bar{x}, \bar{y})$.

Proof. This follows from the combination of Proposition 4.2, Lemma 4.3, and Lemma 4.4. $\triangle$

In the rest of this section we consider bilevel programs with some linearity on the lower level. This issue has been investigated in [10,27], where partial calmness was justified for bilevel programs with linear lower-level problems [27] and with partial linearity imposed only on the $y$-variable [10]. Our goal here is to show that the linearity and partial linearity can be treated by using Theorem 4.5 via the conditions of Lemma 4.4.

To furnish this, we recall first that a cone $A \subset \mathbb{R}^{n}$ is finitely generated if there exists a finite subset $\left\{a_{i}\right\}_{i \in I} \subset A$ such that

$$
A=\left\{\sum_{i \in I} \lambda_{i} a_{i} \mid \lambda_{i} \geq 0\right\}
$$

The following result is classical; its proof can be found, e.g., in the book [2]: $A$ cone in $\mathbb{R}^{n}$ is finitely generated if and only if it is polyhedral. Using this, we derive from Lemma 4.4 that sets of optimal solutions to linear programs are in fact their sets of local weak sharp minimizers.

Lemma 4.6 (local weak sharp minimizers in linear programming). Given $a, b_{i} \in \mathbb{R}^{m}$ and $c_{i} \in \mathbb{R}$ for $i=1, \ldots, p$, consider the linear programming problem:

$$
\text { minimize }\langle a, y\rangle \text { subject to } y \in \Omega:=\left\{y \mid\left\langle b_{i}, y\right\rangle+c_{i} \leq 0 \text { for } i=1, \ldots, p\right\} \text {. }
$$

Then all the assumptions of Lemma 4.4 are satisfied for any optimal solution $\bar{y}$ to (4.10), and thus this problem admits a set of local weak sharp minima at $\tilde{y}$.

Proof. It is well known that the KKT optimality conditions always hold for optimal solutions to linear programs. Thus there exist $\lambda_{i} \geq 0$ as $i \in I(\bar{y})=\left\{i \mid\left\langle b_{i}, \bar{y}\right\rangle+c_{i}=0\right\}$ such that

$$
a+\sum_{i \in I} \lambda_{i} b_{i}=0 \text { with } I=\left\{i \mid \lambda_{i}>0\right\} .
$$

Defining $S:=\left\{y \mid\left\langle b_{i}, y\right\rangle+c_{i}=0, i \in I\right\} \cap \Omega$, we get for all $y \in S$ that

$$
\langle a, \bar{y}\rangle=-\sum_{i \in I} \lambda_{i}\left\langle b_{i}, \bar{y}\right\rangle \geq \sum_{i \in I} \lambda_{i} c_{i}=\langle a, y\rangle
$$


i.e., $S$ is the set of optimal solutions to (4.10). Furthermore, the tangent cone to $\Omega$ at $\vec{y}$ is

$$
T:=T(\bar{y} ; \Omega)=\left\{d \mid\left\langle\hat{b_{i}}, d\right\rangle \leq 0, i \in I(\bar{y})\right\} .
$$

Since all the constraints in (4.10) are linear, the sets $\bar{y}+T$ and $\Omega$ are identical in some neighborhood of $\bar{y}$. Hence we suppose without loss of generality the sets $\Omega=\bar{y}+T=T$ and

$$
S=\left\{\begin{array}{l}
y \mid \begin{array}{l}
\left\langle b_{i}, y\right\rangle+c_{i}=0, i \in I, \\
\left\langle b_{i}, y\right\rangle+c_{i} \leq 0, i \in I(\bar{y}) \backslash I
\end{array}
\end{array}\right\}
$$

are cones, which are finitely generated by the above. Let $d_{1}, \ldots, d_{l}$ be unit vectors generating $\Omega$, let $D:=\left\{d_{j} \mid d_{j} \notin S\right\}$, and let $\eta:=\min \{\langle a, d\rangle, d \in D\}>0$. Then we have

$$
\Omega \subset S+\text { cone } D
$$

i.e., all the assumptions of Lemma 4.4 are satisfied. Thus $S$ is a set of local weak sharp minima at any solution point of the linear program (4.10).

In this way we arrive at the following result in bilevel programming.

Theorem 4.7 (bilevel programs with linear lower levels). Consider the bilevel program:

$$
\text { minimize } F(x, y) \text { subject to } x \in \Omega \subset \mathbb{R}^{n}, y \in S(x) \subset \mathbb{R}^{m} \text {, }
$$

where $S(x)$ is the set optimal solutions to the parametric linear lower-level problem:

$$
\text { minimize }\langle a(x), y\rangle \text { subject to }\left\langle a_{i}, x\right\rangle+\left\langle b_{i}, y\right\rangle+c_{i} \leq 0, i=1, \ldots, p,
$$

where $a_{i} \in \mathbb{R}^{n}, b_{i} \in \mathbb{R}^{m}, c_{i} \in \mathbb{R}$, and the function $a(\cdot): \mathbb{R}^{n} \rightarrow \mathbb{R}^{m}$ is continuous. Then the bilevel program under consideration admits a set of uniformly local weak sharp minima as in (4.6), and hence it is partially calm at any solution point $(\bar{x}, \bar{y})$.

Proof. For every solution $y \in S(x)$ to the parametric lower problem (4.12), find by Lemma 4.6 the corresponding sets $\Omega_{x}$ and $D_{x}$ such that

$$
\Omega_{x} \subset S(x)+\operatorname{cone} D_{x} \text { and } \eta_{x}=\min \left\{\langle a(x), d\rangle \mid d \in D_{x}\right\}>0 .
$$

By the discussion above there are finitely many possible choices of the tangents $T\left(y ; \Omega_{x}\right)$ and hence of $D_{x}$. Considering now $x$ from a neighborhood $U$ of $\vec{x}$ where the function $a(x)$ is sufficiently close to $a(\bar{x})$ by the assumed continuity, we get

$$
\eta:=\inf _{x \in U} \eta_{x}>0
$$

which implies by Lemma 4.4 that the moving set $S(x)$ satisfies all the assumptions of Theorem 4.5. Thus the bilevel program (4.11) is partially calm at its any optimal solution.

Observe that we can similarly proceed with bilevel programs whose lower-level problems are linearly only with respect to $y$. Also the results of Theorem 4.5 can be extended to problems with nonsmooth Lipschitzian data in terms of the limiting subdifferential (2.9). 


\section{Convexified Calculus and Subgradients of Marginal Functions}

The main goal of this section is to establish refined upper estimates for the convexified subdifferential (2.11) of marginal functions that are significant for deriving new necessary optimality conditions for optimistic bilevel programs in what follows. We also obtain some related calculus results needed to achieve this goal and important also for their own sake.

We begin with considering a general class of the marginal functions $\mu(x)$ with the cost function $\varphi(x, y)$ and the constraint mapping $G(x)$ defined in (1.7) in our standing setting of reflexive Banach spaces. Recall [16] that a mapping $S: X \rightrightarrows Y$ is $\mu$-inner semicontinuous at some point $(\bar{x}, \bar{y}) \in \operatorname{gph} S$ if for every sequence $x_{k} \rightarrow \bar{x}$ with $\mu\left(x_{k}\right) \rightarrow \mu(\bar{x})$ there is a sequence of $y_{k} \in S\left(x_{k}\right)$ that contains a subsequence converging to $\bar{y}$. It surely holds for any function $\mu$ if $S$ is inner semicontinuous at $(\vec{x}, \vec{y})$ but not vice versa. The following theorem provides an upper estimate for the convexified subdifferential of the general marginal function (1.7).

Theorem 5.1 (convexified subdifferential of general marginal functions). Let the marginal function $\mu: X \rightarrow \bar{R}$ be given in (1.7) with the corresponding solution map $S: X \rightrightarrows Y$ defined in (1.3), let $(\bar{x}, \bar{y}) \in \operatorname{dom} S$, and let the cost function $\varphi$ in (1.7) be l.s.c. around $(\bar{x}, \bar{y})$ while the constraint mapping $G$ is closed-graph around this point. Assume that either $\varphi$ is SCNEC at $(\bar{x}, \bar{y})$ or $G$ is $S C N C$ at this point and that $S$ is $\mu$-inner semicontinuous at $(\bar{x}, \bar{y})$. Impose in addition the following qualification condition:

$$
\bar{\partial}^{\infty} \varphi(\bar{x}, \bar{y}) \cap(-\bar{N}((\bar{x}, \bar{y}) ; \operatorname{gph} G))=\{0\} .
$$

Then we have the inclusion

$$
\bar{\partial} \mu(\bar{x}) \subset\left\{u^{*} \in X^{*} \mid\left(u^{*}, 0\right) \in \bar{\partial} \varphi(\bar{x}, \bar{y})+\bar{N}((\bar{x}, \bar{y}) ; \operatorname{gph} G)\right\},
$$

which is equivalently written in the coderivative form

$$
\bar{\partial} \mu(\bar{x}) \subset \bigcup_{\left(x^{*}, y^{*}\right) \in \bar{\partial} \varphi(\bar{x}, \bar{y})}\left\{x^{*}+\bar{D}^{*} G(\bar{x}, \bar{y})\left(y^{*}\right)\right\} .
$$

Proof. Define the set

$$
\Lambda:=\left\{u^{*} \in X^{*} \mid\left(u^{*}, 0\right) \in \bar{\partial} \varphi(\bar{x}, \bar{y})+\bar{N}((\bar{x}, \bar{y}) ; \operatorname{gph} G)\right\}
$$

and check that this set is closed in the norm topology of $X^{*}$. Indeed, for a sequence $u_{k}^{*} \rightarrow u^{*}$ as $k \rightarrow \infty$ for some $u^{*} \in X^{*}$ we have by (5.4) that

$$
\left(u_{k}^{*}, 0\right)=\left(x_{1 k}^{*}, y_{1 k}^{*}\right)+\left(x_{2 k}^{*}, y_{2 k}^{*}\right)
$$

with $\left(x_{1 k}^{*}, y_{1 k}^{*}\right) \in \bar{\partial} \varphi(\bar{x}, \bar{y})$ and $\left(x_{2 k}^{*}, y_{2 k}^{*}\right) \in \bar{N}((\bar{x}, \bar{y}) ; \operatorname{gph} G)$ for all $k \in \mathbb{N}$. We show first that the sequence $\left\{z_{k}^{*}:=\left(x_{1 k}^{*}, y_{1 k}^{*}\right)\right\}$ is bounded in $X^{*} \times Y^{*}$. Suppose on the contrary that $\left\|z_{k}^{*}\right\| \rightarrow \infty$ and get from (5.5) that

$$
\frac{1}{\left\|z_{k}^{*}\right\|}\left(u_{k}^{*}, 0\right)=\frac{z_{k}^{*}}{\left\|z_{k}^{*}\right\|}+\left(u_{k}^{*}, v_{k}^{*}\right) \text { with }\left(u_{k}^{*}, v_{k}^{*}\right)=\frac{1}{\left\|z_{k}^{*}\right\|}\left(x_{2 k}^{*}, y_{2 k}^{*}\right)
$$

with some $v_{k}^{*} \in Y^{*}$ for all $k \in \mathbb{N}$ sufficiently large. On the other hand, we have

$$
\left.\left(\frac{z_{k}^{*}}{\left\|z_{k}^{*}\right\|}, \frac{-1}{\left\|z_{k}^{*}\right\|}\right) \in \bar{N}((\bar{x}, \bar{y}), \varphi(\bar{x}, \bar{y})) ; \operatorname{epi} \varphi\right)
$$


By extracting a subsequence without relabeling and loss of generality, assume that

$$
\left.\left(u_{k}^{*}, v_{k}^{*}\right) \stackrel{w}{\rightarrow}\left(u^{*}, v^{*}\right) \text { and }\left(\frac{z_{k}^{*}}{\left\|z_{k}^{*}\right\|}, \frac{-1}{\left\|z_{k}^{*}\right\|}\right) \stackrel{w}{\rightarrow}\left(z^{*}, 0\right) \in \bar{N}((\bar{x}, \bar{y}), \varphi(\bar{x}, \bar{y})) ; \operatorname{epi} \varphi\right)
$$

with some $v^{*} \in Y^{*}$ and $k \rightarrow \infty$. It follows that $z^{*} \in \bar{\partial}^{\infty} \varphi(\bar{x}, \bar{y})$ and

$$
0=z^{*}+\left(u^{*}, v^{*}\right) \in \bar{\partial}^{\infty} \varphi(\bar{x}, \bar{y})+\bar{N}((\bar{x}, \bar{y}) ; \operatorname{gph} G),
$$

which implies therefore that $z^{*}=0$ and $\left(u^{*}, v^{*}\right)=(0,0)$ by the qualification condition (5.1). If $\varphi$ is SCNEC at $(\bar{x}, \bar{y})$, we conclude that $\frac{z_{k}^{*}}{\left\|z_{k}^{*}\right\|} \rightarrow 0$, a contradiction. Similarly we arrive at a contradiction assuming alternatively that $G$ is SCNC at $(\bar{x}, \bar{y})$ and thus justify the boundedness of the sequence $\left\{\left(x_{1 k}^{*}, y_{1 k}^{*}\right)\right\}$ in $X^{*} \times Y^{*}$. The latter ensures that there is a convergent subsequence $\left(x_{1 k}^{*}, y_{1 k}^{*}\right) \stackrel{w}{\rightarrow}\left(\widetilde{x}_{1}^{*}, \widetilde{y}_{1}^{*}\right)$ for some pair $\left(\widetilde{x}_{1}^{*}, \widetilde{y}_{1}^{*}\right)$, which belongs to the convexified subdifferential $\bar{\partial} \varphi(\bar{x}, \bar{y})$ as $k \rightarrow \infty$ due to the weak closedness of this convex set. Further, it follows from (5.5) due to the norm convergence of $u_{k}^{*}$ that the sequence $\left\{\left(x_{2 k}^{*}, y_{2 k}^{*}\right)\right\}$ weakly converges in $X^{*} \times Y^{*}$ to some element $\left(\widetilde{x}_{2}^{*}, \widetilde{y}_{2}^{*}\right)$ belonging to the convex set $\left.\bar{N}((\bar{x}, \bar{y}) ; \operatorname{gph} G)\right)$ by the arguments above. Passing to the limit in (5.5) gives us $\left(u^{*}, 0\right)=\left(\widetilde{x}_{1}^{*}, \widetilde{y}_{1}^{*}\right)+\left(\widetilde{x}_{2}^{*}, \widetilde{y}_{2}^{*}\right)$ and thus verifies that $u^{*} \in \Lambda$, which ensures that the latter set is closed.

The next step in the proof of the theorem is to justify the validity of the inclusion

$$
\partial \mu(\bar{x})+\partial^{\infty} \mu(\bar{x}) \subset \Lambda
$$

involving the limiting subdifferential (2.9) and its singular counterpart (2.10). To proceed, fix any $u^{*}=u_{1}^{*}+u_{2}^{*}$ with $u_{1}^{*} \in \partial \mu(\bar{x})$ and $u_{2}^{*} \in \partial^{\infty} \mu(\bar{x})$. By the proof of [14, Theorem 1.108] (given under the inner semicontinuity assumption on $S$ while it holds under the weaker $\mu$-inner semicontinuity one; cf. [16, Theorem 5.1] for the singular subdifferential case) we have

$$
\begin{gathered}
\partial \mu(\bar{x}) \subset\left\{u^{*} \mid\left(u^{*}, 0\right) \in \partial(\varphi+\delta(\cdot, \operatorname{gph} G))\right\}, \\
\partial^{\infty} \mu(\bar{x}) \subset\left\{u^{*} \mid\left(u^{*}, 0\right) \in \partial^{\infty}(\varphi+\delta(\cdot, \operatorname{gph} G))\right\} .
\end{gathered}
$$

It follows from our assumptions that the qualification condition (5.1) holds and that either $\varphi$ is SNEC at $(\bar{x}, \bar{y})$ or $G$ is SNC at this point. Employing now the subdifferential sum rules from [14, Theorem 3.36] to (5.7) and (5.8) gives us

$$
\begin{aligned}
\left(u^{*}, 0\right)=\left(u_{1}^{*}, 0\right)+\left(u_{2}^{*}, 0\right) & \in[\partial \varphi(\bar{x}, \bar{y})+N((\bar{x}, \bar{y}) ; \operatorname{gph} G)]+\left[\partial^{\infty} \varphi(\bar{x}, \bar{y})+N((\bar{x}, \bar{y}) ; \operatorname{gph} G)\right] \\
& \subset \operatorname{clco}\left[\partial \varphi(\bar{x}, \bar{y})+\partial^{\infty} \varphi(\bar{x}, \bar{y})\right]+\bar{N}((\bar{x}, \bar{y}) ; \operatorname{gph} G) \\
& =\bar{\partial} \varphi(\bar{x}, \bar{y})+\bar{N}((\bar{x}, \bar{y}) ; \operatorname{gph} G),
\end{aligned}
$$

which ensures that $\partial \mu(\bar{x})+\partial^{\infty} \mu(\bar{x}) \subset \Lambda$ and thus the claimed inclusion (5.2) by (2.11), (5.4), and the proved closedness and convexity of the latter set. Finally, the coderivative representation (5.3) readily follows from (5.2) by definition (2.7).

In the subsequent analysis of this section we intend to obtain a constructive upper estimate for the convexified subdifferential of the marginal function (1.7) for the bilevel program under consideration, where the constraint mapping $G$ in the lower-level problem is described by inequalities (4.2) as well as in more general cases. To furnish this, we need to derive some calculus rules that are certainly of their own interest.

The first calculus results gives and exact formula and an upper estimate for the convexified normal cone of inverse images of sets under appropriate assumptions in infinite dimensions; $\mathrm{cf}$. [21, Theorem 7.1] and [22, Exercise 6.7] for their finite-dimensional versions. 
Theorem 5.2 (convexified normals to inverse images of sets under strictly differentiable mappings). Let $f: X \rightarrow Y$ be strictly differentiable at $\bar{x}$, and let $\Theta \subset Y$ with $\bar{y}:=f(\bar{x}) \in \Theta$. The following assertions hold:

(i) If the derivative $\nabla f(\bar{x}): X \rightarrow Y$ is surjective, then

$$
\bar{N}\left(\widetilde{x} ; f^{-1}(\Theta)\right)=\nabla f(\bar{x})^{*} \bar{N}(\bar{y} ; \Theta) .
$$

(ii) Suppose that $\Theta$ is locally closed around $\bar{y}$ and $S C N C$ at this point and that

$$
\operatorname{ker} \nabla f(\bar{x})^{*} \cap \bar{N}(\bar{y} ; \Theta)=\{0\} .
$$

Then we have the inclusion

$$
\bar{N}\left(\bar{x} ; f^{-1}(\Theta)\right) \subset \nabla f(\bar{x})^{*} \bar{N}(\bar{y} ; \Theta),
$$

which holds as equality provided that $\Theta$ is normally regular at $\bar{y}$.

Proof. To justify (i), apply [14, Theorem 1.17] and get

$$
N\left(\bar{x} ; f^{-1}(\Theta)\right)=\nabla f(\bar{x})^{*} N(\bar{y} ; \Theta) \subset \nabla f(\bar{x})^{*} \bar{N}(\bar{y} ; \Theta) .
$$

Since $\bar{N}(\bar{y} ; \Theta)$ is convex, the set $\nabla f(\bar{x})^{*} \bar{N}(\bar{y} ; \Theta)$ is convex as well. Let us show that this set is closed in the norm topology of $X^{*}$. Indeed, fix any sequence $x_{k}^{*} \rightarrow x^{*}$ with $x_{k}^{*} \in \nabla f(\bar{x})^{*} \bar{N}(\bar{y} ; \Theta)$ and take $y_{k}^{*} \in \bar{N}(\bar{y} ; \Theta)$ with $x_{k}^{*}=\nabla f(\bar{x})^{*} y_{k}^{*}$ for all $k \in \mathbb{N}$. It follows from the surjectivity of $\nabla f(\bar{x})$ that there is $\kappa>0$ such that

$$
\left\|x_{k}^{*}-x_{m}^{*}\right\|=\left\|\nabla f(\bar{x})^{*} y_{k}^{*}-\nabla f(\bar{x})^{*} y_{m}^{*}\right\| \geq \kappa|| y_{k}^{*}-y_{m}^{*} \| \text { whenever } k, m \in \mathbb{N}
$$

This implies that $\left\{y_{k}^{*}\right\}$ is a Cauchy sequence; hence it converges to some $y^{*} \in \bar{N}(\bar{y} ; \Theta)$, which ensures that $x^{*}=\nabla f(\tilde{x})^{*} y^{*} \in \bar{N}(\bar{y} ; \Theta)$ and so the set $f(\bar{x})^{*} \bar{N}(\bar{y} ; \Theta)$ is closed. Thus we have

$$
\bar{N}\left(\bar{x} ; f^{-1}(\Theta)\right) \subset \nabla f(\bar{x})^{*} \bar{N}(\bar{y} ; \Theta) .
$$

The converse inclusion " $\supset$ " follows from (5.12) and the boundedness of the operator $\nabla f(\bar{x})$ by

$$
\begin{aligned}
\nabla f(\bar{x})^{*} \bar{N}(\bar{y} ; \Theta) & =\nabla f(\bar{x})^{*}(\operatorname{clco} N(\bar{y} ; \Theta)) \subset \operatorname{cl} \nabla f(\bar{x})^{*}(\operatorname{co} N(\bar{y} ; \Theta)) \\
& \subset \operatorname{clco} \nabla f(\bar{x})^{*} N(\bar{y} ; \Theta)=\operatorname{clco} N\left(\bar{x} ; f^{-1}(\Theta)\right)=\bar{N}\left(\bar{x} ; f^{-1}(\Theta)\right) .
\end{aligned}
$$

Next we justify (ii) observing similarly to the proof of (i) that it remains showing the closedness of the set $\nabla f(\bar{x})^{*} \bar{N}(\bar{y} ; \Theta)$ in order to the ensure the validity of (5.11). To proceed, take an arbitrary sequence $x_{k}^{*} \rightarrow x^{*}$ with $x_{k}^{*}=\nabla f(\bar{x})^{*} y_{k}^{*}$ for some $y_{k}^{*} \in \bar{N}(\bar{y} ; \Theta)$ as $k \in \mathbb{N}$. Let us verify that the sequence $\left\{y_{k}^{*}\right\}$ is bounded in $Y^{*}$. Assume the contrary and get with no loss of generality that $\left\|y h_{k}^{*}\right\| \rightarrow \infty$ as $k \rightarrow \infty$. Then denote

$$
z_{k}^{*}:=\frac{x_{k}^{*}}{\left\|y_{k}^{*}\right\|}=\nabla f(\vec{x})^{*}\left(\frac{y_{k}^{*}}{\left\|y_{k}^{*}\right\|}\right), \quad k \in \mathbb{N}
$$

by extracting a subsequence if necessary, that $z_{k}^{*} \stackrel{w}{\rightarrow} z^{*} \in N_{C}(\bar{y} ; \Theta)$ for some $z^{*} \in Y^{*}$. Thus $0=$ $\nabla f(\bar{x})^{*} z^{*}$, which implies by the qualification condition (5.10) that $z^{*}=0$. Invoking the assumed SCNC property of $\Theta$ at $\bar{y}$, we get $\left\|z_{k}^{*}\right\| \rightarrow 0$, which is a contradiction. Thus the sequence $\left\{y_{k}^{*}\right\}$ is bounded, which ensures the existence of $y^{*} \in \bar{N}(\bar{y} ; \Theta)$ such that $y_{k}^{*} \stackrel{w}{\rightarrow} y^{*}$ along a subsequence of $k \rightarrow \infty$. This gives $x^{*}=\nabla f(\bar{x})^{*} y^{*} \in \nabla f(\bar{x})^{*} \bar{N}(\bar{y} ; \Theta)$ and justifies inclusion (5.11).

Finally, the normal regularity of $\Theta$ at $\bar{y}$ implies that

$$
\nabla f(\bar{x})^{*} \bar{N}(\bar{y} ; \Theta)=\nabla f(\bar{x})^{*} \widehat{N}(\bar{y} ; \Theta) \subset \widehat{N}\left(\bar{x} ; f^{-1}(\Theta)\right) \subset \bar{N}\left(\bar{x} ; f^{-1}(\Theta)\right)
$$


which ensures the equality in (5.11) in this case and completes the proof of the theorem.

Theorem 5.2 has a number of important consequences and applications. We present below those related to our analysis of marginal functions in the parametric optimization problems under consideration. First consider the parametric constraint system given by

$$
G(x):=\{y \in Y \mid g(x, y) \in \Theta\},
$$

with a single-valued mapping $g: X \times Y \rightarrow Z$ between reflexive Banach spaces and evaluate via Theorem 5.2 the convexified coderivative of the set-valued mapping $F: X \rightrightarrows Y$ from (5.13).

Corollary 5.3 (convexified coderivative of constraint systems). Let $(\bar{x}, \bar{y}) \in \operatorname{gph} G$ with $G$ given in (5.13), and let $\bar{z}:=g(\bar{x}, \bar{y}) \in \Theta$. Assume that $g$ is strictly differentiable at $(\bar{x}, \bar{y})$. Then the following assertions hold:

(i) If $\nabla g(\bar{x}, \bar{y})$ is surjective, then

$$
\bar{D}^{*} G(\bar{x}, \bar{y})\left(y^{*}\right)=\left\{x^{*} \in X^{*} \mid\left(x^{*},-y^{*}\right) \in \nabla g(\bar{x}, \bar{y})^{*} \bar{N}(\bar{z} ; \Theta)\right\} \text { for all } y^{*} \in Y^{*} .
$$

(ii) Let the set $\Theta$ be locally closed around $\bar{z}$ with the $S C N C$ property at this point, and let the qualification condition

$$
\bar{N}(\bar{z} ; \Theta) \cap \operatorname{ker} \nabla g(\bar{x}, \bar{y})^{*}=\{0\}
$$

is satisfied. Then we have

$$
\bar{D}^{*} G(\bar{x}, \bar{y})\left(y^{*}\right) \subset\left\{x^{*} \in X^{*} \mid\left(x^{*},-y^{*}\right) \in \nabla g(\bar{x}, \bar{y})^{*} \bar{N}(\bar{z} ; \Theta)\right\},
$$

where the equality holds when $\Theta$ is normally regular at $\bar{z}$.

Proof. Observing that $\operatorname{gph} G=g^{-1}(\Theta)$, we derive this result from Theorem 5.2.

Next we present a specification of Corollary 5.3 for the parametric constraint systems arising in nonlinear programming with equality and inequality constraints given by differentiable functions.

Corollary 5.4 (convexified coderivative of feasible solution maps in nonlinear programming). Let $G: X \rightrightarrows Y$ be given by

$$
\begin{aligned}
G(x):=\{y \in Y \mid & g_{i}(x, y) \leq 0 \text { for } i=1, \ldots, p, \\
& \left.g_{i}(x, y)=0 \text { for } i=p+1, \ldots, p+r\right\},
\end{aligned}
$$

where all the functions $g_{i}$ are strictly differentiable at $(\bar{x}, \bar{y}) \in \operatorname{gph} G$. Define the index set

$$
I(\bar{x}, \bar{y}):=\left\{i \in\{1, \ldots, p+r\} \mid g_{i}(\bar{x}, \bar{y})=0\right\}
$$

and assume the fulfillment of the Mangasarian-Fromovitz constraint qualification:

(a) the gradients $\nabla g_{p+1}(\bar{x}, \bar{y}), \ldots, \nabla g_{p+r}(\bar{x}, \bar{y})$ are linearly independent;

(b) there is $w \in X \times Y$ such that $\left\langle\nabla g_{i}(\bar{x}, \bar{y}), w\right\rangle=0$ for $i=p+1, \ldots, p+r$ and that $\left\langle\nabla g_{i}(\bar{x}, \bar{y}), w\right\rangle<0$ whenever $i=1, \ldots, p$ with $g_{i}(\bar{x}, \bar{y})=0$.

Then we have the coderivative representation

$$
\begin{aligned}
\bar{D}^{*} G(\bar{x}, \bar{y}):=\left\{x^{*} \in X^{*} \mid\right. & \left(x^{*},-y^{*}\right)=\sum_{i \in I(\bar{x}, \bar{y})} \lambda_{i} \nabla g_{i}(\bar{x}, \bar{y}), \\
& \left.\lambda_{i} \geq 0 \text { for } i \in\{1, \ldots, p\} \cap I(\bar{x}, \bar{y})\right\} .
\end{aligned}
$$


Proof. Employ Corollary 5.3 with $g:=\left(g_{1}, \ldots, g_{p+r}\right)$ and $\Theta$ given by

$$
\Theta:=\left\{\left(\alpha_{1}, \ldots, \alpha_{p+r}\right) \in \mathbb{R}^{p+r} \mid \begin{array}{l}
\alpha_{i} \leq 0 \text { for } i=1, \ldots, p, \\
\left.\alpha_{i}=0 \text { for } i=p+1, \ldots, p+r\right\} .
\end{array}\right.
$$

Denoting $\bar{z}:=g(\bar{x}, \bar{y})$ and taking into account that the set $\Theta$ is convex, we get

$$
N(\bar{z} ; \Theta)=\left\{\left(\lambda_{1}, \ldots, \lambda_{p+r}\right) \in \mathbb{R}^{p+r} \mid \lambda_{i} \geq 0, \lambda_{i} g_{i}(\bar{x}, \bar{y})=0 \text { for } i=1, \ldots, p\right\} .
$$

Observe that the Mangasarian-Fromovitz constraint qualification (5.17) corresponds to the validity of the qualification condition (5.14) of Corollary 5.3, and thus the coderivative representation (5.18) follows directly from the equality in (5.15).

Substituting the coderivative representations obtained in Corollaries 5.3 and 5.4 into inclusion (5.3) of Theorem 5.3 gives us, under the corresponding qualification conditions, upper estimates of the convexified subdifferential of the marginal function.

Let us present the result in this direction for the case of nonlinear programming systems (5.16). To proceed, consider the set of Lagrange (KKT) multipliers in the the problem of minimizing $\varphi(x, y)$ subject to the constraints in (5.16) defined by

$$
\begin{aligned}
\Lambda(\bar{x}, \bar{y}):=\left\{\lambda \in \mathbb{R}^{p+r} \mid\right. & \nabla_{y} g(\bar{x}, \bar{y})+\sum_{i=1}^{p+r} \lambda_{i} \nabla_{y} g_{i}(\bar{x}, \bar{y})=0, \\
& \left.\lambda_{i} \geq 0, \lambda_{i} g_{i}(\bar{x}, \bar{y})=0 \text { for } i=1, \ldots, p\right\} .
\end{aligned}
$$

Corollary 5.5 (convexified subdifferential of marginal functions in nonlinear programming). Let the constraint mapping $G$ in (1.7) be given by (5.16), and let the functions $\varphi$ and $g_{i}$ be strictly differentiable at $(\bar{x}, \bar{y}) \in \mathrm{gph} G$. Assume that the Mangasarian-Fromovitz constraint qualification (5.17) is satisfied and the solution map $S$ in (1.3) is $\mu$-inner semicontinuous at $(\bar{x}, \bar{y})$. Then we have the inclusion

$$
\bar{\partial} \mu(\bar{x}) \subset \bigcup_{\lambda \in \Lambda(\bar{x}, \bar{y})}\left[\nabla_{x} \varphi(\bar{x}, \bar{y})+\sum_{i=1}^{p+r} \lambda_{i} \nabla_{x} g_{i}(\bar{x}, \bar{y})\right],
$$

where the set of multipliers $\Lambda(\bar{x}, \bar{y})$ is defined in (5.19).

Proof. This follows directly from Theorem 5.1 and Corollary 5.4 due to the facts that

$$
\bar{\partial} \varphi(\bar{x}, \bar{y})=\bar{\partial}_{x} \varphi(\bar{x}, \bar{y}) \times \bar{\partial}_{y} \varphi(\bar{x}, \bar{y}), \quad \bar{\partial} \varphi(\bar{x}, \bar{y})=\{\nabla \varphi(\bar{x}, \bar{y})\}, \quad \bar{\partial}^{\infty} \varphi(\bar{x}, \bar{y})=\{0\}
$$

and $\varphi$ is SCNEC at $(\bar{x}, \bar{y})$ for any function $\varphi$ strictly differentiable at $(\bar{x}, \bar{y})$.

Remark 5.6 (comparison with known upper estimates for subdifferentials of marginal functions). The results obtained in Theorem 5.1 and Corollary 5.5 (see also Theorem 5.9 below) are different from known ones for the convexified/Clarke subdifferentials of marginal functions established with no inner semicontinuity, while with other, assumptions imposed on the solution map (1.3); see, e.g., [5, 14, 21, 22, 27] and the references therein. The main point is that they do not require additional convexification procedures over solution maps, which significantly enlarges the corresponding upper estimates and leads to weaker necessary optimality conditions for bilevel programs even in the case of smooth initial data; see Section 6 for more details.

Next we intend to derive an upper estimate for the convexified subdifferential of the marginal function (1.7) in the case of nonsmooth constraints in (4.2). To proceed, obtain first the following intersection rule for the convexified normal cone (2.4) in infinite dimensions, which extends the corresponding finite-dimensional result of [21, Corollary 8.1.1] and is definitely of its own interest. 
Theorem 5.7 (intersection rule for convexified normals). Let $\Omega_{1}$ and $\Omega_{2}$ be closed subsets of $X$ with $\vec{x} \in \Omega_{1} \cap \Omega_{2}$. Suppose that at least one of the sets $\Omega_{1}, \Omega_{2}$ is SCNC at $\bar{x}$ and that the normal qualification condition

$$
\bar{N}\left(\bar{x} ; \Omega_{1}\right) \cap\left[-\bar{N}\left(\bar{x} ; \Omega_{2}\right)\right]=\{0\}
$$

is satisfied. Then we have the inclusion

$$
\dot{\bar{N}}\left(\bar{x} ; \Omega_{1} \cap \Omega_{2}\right) \subset \bar{N}\left(\bar{x} ; \Omega_{1}\right)+\bar{N}\left(\bar{x} ; \Omega_{2}\right),
$$

which holds as equality if both sets $\Omega_{1}$ and $\Omega_{2}$ are normally regular at $\bar{x}$; in this case the intersection set $\Omega_{1} \cap \Omega_{2}$ is normally regular at $\bar{x}$ as well.

Proof. It follows from [14, Corollary 3.37] that

$$
N\left(\bar{x} ; \Omega_{1} \cap \Omega_{2}\right) \subset N\left(\bar{x} ; \Omega_{1}\right)+N\left(\bar{x} ; \Omega_{2}\right) \subset \bar{N}\left(\bar{x} ; \Omega_{2}\right)+\bar{N}\left(\bar{x} ; \Omega_{2}\right)
$$

under the qualification condition (5.21). Since the set on the right-hand side above is obviously convex, to get (5.22) by (2.4) it remains to show that this set is closed. To proceed, fix a sequence $x_{k}^{*} \in \bar{N}\left(\bar{x} ; \Omega_{1}\right)+\bar{N}\left(\bar{x} ; \Omega_{2}\right)$, which converges to $x^{*}$ as $k \rightarrow \infty$, and hence the sequence $\left\{x_{k}^{*}\right\}$ by the uniform boundedness principle. For each $k \in \mathbb{N}$ there exist $u_{k}^{*} \in \bar{N}\left(\bar{x} ; \Omega_{1}\right)$ and $v_{k}^{*} \in \mathbb{B} N\left(\bar{x} ; \Omega_{2}\right)$ such that $x_{k}^{*}=u_{k}^{*}+v_{k}^{*}$. Assuming for definiteness that the set $\Omega_{1}$ is SCNC at $\bar{x}$, we conclude that the sequence $\left\{u_{k}^{*}\right\}$ is bounded in $X^{*}$. Indeed, suppose the contrary and get with no loss of generality that $\left\|u_{k}^{*}\right\| \rightarrow \infty$ as $k \rightarrow \infty$. Then

$$
\frac{x_{k}^{*}}{\left\|u_{k}^{*}\right\|}=z_{k}^{*}+\frac{v_{k}^{*}}{\left\|u_{k}^{*}\right\|} \text { with } z_{k}^{*}:=\frac{u_{k}^{*}}{\left\|u_{k}^{*}\right\|},
$$

and thus $z_{k}^{*} \stackrel{w}{\rightarrow} z^{*} \in \bar{N}\left(\bar{x} ; \Omega_{1}\right)$ along a subsequence of $k \in \mathbb{N}$. It follows from (5.23) that

$$
\frac{v_{k}^{*}}{\left\|u_{k}^{*}\right\|} \stackrel{w}{\rightarrow}-z^{*} \in \bar{N}\left(\bar{x} ; \Omega_{2}\right) \text { as } k \rightarrow \infty,
$$

which implies by the qualification condition (5.21) that $z^{*}=0$. By the assumed SCNC property of $\Omega_{1}$ at $\bar{x}$ we have $\left\|z_{k}^{*}\right\| \rightarrow 0$, which is impossible due to $\left\|z_{k}^{*}\right\|=1$. Thus the sequence $\left\{u_{k}^{*}\right\}$ is bounded and so is $\left\{v_{k}^{*}\right\}$. This implies the existence of $u^{*} \in \bar{N}\left(\bar{x} ; \Omega_{1}\right)$ and $v^{*} \in \bar{N}\left(\bar{x} ; \Omega_{2}\right)$ such that $u_{k}^{*} \stackrel{w}{\rightarrow} u^{*}$ and $v_{k}^{*} \stackrel{w}{\rightarrow} v^{*}$ along a subsequence of $k \in \mathbb{N}$. Therefore we have $x^{*}=u^{*}+v^{*} \in \bar{N}\left(\bar{x} ; \Omega_{1}\right)+\bar{N}\left(\bar{x} ; \Omega_{2}\right)$ and so justify (5.22). The opposite inclusion to (5.22) and the normal regularity of $\Omega_{1} \cap \Omega_{2}$ follows, under the normal regularity assumptions on $\Omega_{1}$ and $\Omega_{2}$, from

$$
\bar{N}\left(\bar{x} ; \Omega_{1}\right)+\bar{N}\left(\bar{x} ; \Omega_{2}\right)=\widehat{N}\left(\bar{x} ; \Omega_{1}\right)+\widehat{N}\left(\bar{x} ; \Omega_{2}\right) \subset \widehat{N}\left(\bar{x} ; \Omega_{1} \cap \Omega_{2}\right) \subset \bar{N}\left(\bar{x} ; \Omega_{1} \cap \Omega_{2}\right) .
$$

This completes the proof of the theorem.

Yet another calculus result is important for its own sake and is useful in what follows.

Proposition 5.8 (convexified normal cone to inequality constraints). Consider the sets

$$
\Omega_{i}=\left\{x \mid g_{i}(x) \leq 0\right\}, \quad i=1, \ldots, p,
$$

where each function $g_{i}$ is locally Lipschitzian around $\bar{x} \in \cap_{i=1}^{p} \Omega_{i}$. Assume the fulfilments of the nonsmooth Mangasarian-Fromovitz constraint qualification:

$$
\left[\sum_{i \in I(\bar{x})} \lambda_{i} x_{i}^{*}=0 \text { with } x_{i}^{*} \in \bar{\partial} g_{i}(\bar{x}), \lambda_{i} \geq 0\right] \Longrightarrow \lambda_{i}=0 \text { for } i \in I(\bar{x}),
$$


where $I(\bar{x})$ is the collection of active constraint indices at $\bar{x}$. Then for any convexified normal $x^{*} \in \bar{N}\left(\bar{x} ; \bigcap_{i=1}^{p} \Omega_{i}\right)$ we have the inclusion

$$
x^{*} \in \sum_{i \in I(\bar{x})} \lambda_{i} \bar{\partial} g_{i}(\bar{x}) \text { with some } \lambda_{i} \geq 0
$$

Proof. Observe first that for any function $\varphi: X \rightarrow \bar{R}$ locally Lipschitzian around $\bar{x}$ we have

$$
\bar{N}(\bar{x}, \Omega) \subset \bigcup_{\lambda \geq 0} \lambda \bar{\partial} \varphi(\bar{x}) \text { with } \Omega:=\{x \in X \mid \varphi(x) \leq 0\} .
$$

provided that $0 \notin \bar{\partial} \varphi(\bar{x})$. It is sufficient to prove it for $\varphi(\bar{x})=0$. To proceed, consider the set $O:=X \times\{0\} \subset X \times \mathbb{R}$ for which $\Omega \times\{0\}=\operatorname{epi} \varphi \cap O$. Let us check that

$$
\bar{N}((\bar{x}, 0) ; \operatorname{epi} \varphi) \cap[-\bar{N}((\bar{x}, 0) ; O)]=\{0\},
$$

i.e., the qualification condition (5.21) holds for the sets epi $\varphi$ and $O$. Indeed, take $\left(x^{*},-\lambda\right)$ from the set on left-hand side of (5.28) and observe from the structure of $O$ that $x^{*}=0$. It follows from $\left(x^{*},-\lambda\right) \in \bar{N}((\bar{x}, 0) ;$ epi $\varphi)$ that $\lambda \geq 0$. If $\lambda>0$, then

$$
\frac{1}{\lambda}\left(x^{*},-\lambda\right)=(0,-1) \in \bar{N}((\bar{x}, 0) ; \text { epi } \varphi) .
$$

Hence $0 \in \bar{\partial} \varphi(\bar{x})$, a contradiction that justifies (5.28). Applying now Theorem 5.7 gives us

$$
\bar{N}(\bar{x}, \Omega) \times \mathbb{R}=\bar{N}((\bar{x}, 0) ; \Omega \times\{0\}) \subset \bar{N}((\bar{x}, 0) ; \operatorname{epi} \varphi)+\{0\} \times \mathbb{R},
$$

which readily implies the inclusion in (5.27).

To continue the proof of (5.26), note that the qualification condition (5.25) yields that $0 \notin$ $\bar{\partial} g_{i}(\bar{x})$ for all $i \in I(\bar{x})$, and thus we have by (5.24) and (5.27) that

$$
\bar{N}\left(\bar{x}, \Omega_{i}\right) \subset \bigcup_{\lambda \geq 0} \lambda \bar{\partial} g_{i}(\bar{x}) \text { for all } i \in I(\bar{x})
$$

while $\bar{N}\left(\bar{x}, \Omega_{i}\right)=\{0\}$ if $i \notin I(\bar{x})$. Now using (5.25) and Theorem 5.7, we get

$$
\bar{N}\left(\bar{x} \bigcap_{i=1}^{p} \Omega_{i}\right) \subset \bar{N}\left(\bar{x} ; \Omega_{1}\right)+\bar{N}\left(\bar{x} ; \Omega_{2}\right)+\ldots+\bar{N}\left(\bar{x} ; \Omega_{p}\right) .
$$

Indeed, this inclusion holds for $p=2$ by Theorem 5.7 and can be easily derived in the general case by induction involving the qualification conditions (5.10) and (5.25). The claimed result (5.26) now follows from inclusions (5.27) and (5.29).

Now we are ready to estimate the convexified subdifferential of the marginal function (1.7) with the constraint mapping $G$ defined by inequalities as in (1.2).

Theorem 5.9 (convexified subdifferential of marginal functions with nonsmooth inequality constraints). Let the marginal function $\mu$ be defined in (1.7) with the constraint mapping $G$ given in (1.2). Assume that $\varphi$ and $g_{i}$ are Lipschitz around $(\bar{x}, \bar{y}) \in \operatorname{gph} G$ and that the qualification condition (5.25) is satisfied for $g_{i}$ at $(\vec{x}, \bar{y})$. Suppose also that the corresponding solution map $S$ in $(1.3)$ is inner semicontinuous at $(\bar{x}, \bar{y})$. Then we have the inclusion

$$
\bar{\partial} \mu(\bar{x}) \subset \bigcup\left\{x^{*} \in X^{*} \mid\left(x^{*}, 0\right) \in \bar{\partial} \varphi(\bar{x}, \bar{y})+\sum_{i=1}^{p} \lambda_{i} \bar{\partial} g_{i}(\bar{x}, \bar{y}), \lambda_{i} \geq 0, \lambda_{i} g_{i}(\bar{x}, \bar{y})=0\right\} .
$$


Proof. Observe first that

$$
\operatorname{gph} G=\bigcap_{i=1}^{p}\left\{(x, y) \in X \times Y \mid g_{i}(x, y) \leq 0\right\} .
$$

Invoking Proposition 5.8 gives us

$$
\bar{N}((\bar{x}, \bar{y}) ; \operatorname{gph} G) \subset\left\{\sum_{i=1}^{p} \lambda_{i} \bar{\partial} g_{i}(\bar{x}, \bar{y}) \mid \lambda_{i} \geq 0, \lambda_{i} g_{i}(\vec{x}, \bar{y})=0\right\} .
$$

To get (5.30), it remains to apply Theorem 5.1 to the marginal function in question.

\section{$6 \quad$ Necessary Optimality Conditions for Bilevel Programs}

This section is devoted to deriving necessary optimality conditions for the bilevel programs (4.1) with the inequality constraints (4.2) and (4.3) based on the calculus results obtained above. First we recall some qualification conditions needed in what follows using the terminology widely accepted in bilevel programming; see, e.g., $[6,8]$. Denote

$$
I(\bar{x}, \bar{y}):=\left\{i \in\{1, \ldots, p\} \mid g_{i}(\bar{x}, \bar{y})=0\right\} .
$$

Given a point $(\bar{x}, \bar{y}) \in X \times Y$ satisfying the lower-level inequality constraints (4.2) with the active index set $I(\bar{x}, \bar{y})$, we say that $(\bar{x}, \bar{y})$ is lower-level regular if the following implication holds in terms of limiting subgradients:

$$
\left[\sum_{i \in I(\bar{x}, \bar{y})} \lambda_{i} v_{i}^{*}=0, \lambda_{i} \geq 0\right] \Longrightarrow\left[\lambda_{i}=0 \text { for all } i \in I(\bar{x}, \bar{y})\right]
$$

whenever $\left(u_{i}^{*}, v_{i}^{*}\right) \in \partial g_{i}(\bar{x}, \bar{y})$ with some $u_{i}^{*} \in X^{*}$ as $i \in I(\bar{x}, \bar{y})$. Similarly, given $\vec{x} \in X$ satisfying the upper-level inequality constraints (4.3) with the active index set

$$
J(\bar{x}):=\left\{j \in\{1, \ldots, m\} \mid h_{j}(\bar{x})=0\right\},
$$

we say that $\bar{x}$ is upper-level regular if

$$
\left[0 \in \sum_{j \in J(\tilde{x})} \lambda_{j} \partial h_{j}(\bar{x}), \lambda_{j} \geq 0\right] \Longrightarrow\left[\lambda_{j}=0 \text { for all } j \in J(\bar{x})\right] .
$$

Note that for functions $g_{i}$ and $h_{j}$ strictly differentiable at the reference points the lower-level regularity (6.1) and the upper-level regularity reduce, respectively, to

$$
\begin{gathered}
{\left[\sum_{i \in I(\bar{x}, \bar{y})} \lambda_{i} \nabla_{y} g_{i}(\vec{x}, \bar{y})=0, \lambda_{i} \geq 0\right] \Longrightarrow\left[\lambda_{i}=0 \text { for all } i \in I(\bar{x}, \bar{y})\right],} \\
{\left[\sum_{j \in J(\bar{x})} \lambda_{j} \nabla h_{j}(\bar{x})=0, \lambda_{j} \geq 0\right] \Longrightarrow\left[\lambda_{j}=0 \text { for all } j \in J(\bar{x})\right] .}
\end{gathered}
$$

First we derive optimality necessary conditions for bilevel programs with strictly differentiable data. It follows from the proof below that the result holds for problems with equality constraints on both lower and upper levels. 
Theorem 6.1 (necessary optimality conditions for smooth bilevel programs, $\mathrm{I}$ ). Let $(\bar{x}, \bar{y})$ be a local optimal solution to the constrained bilevel program (4.1)-(4.3). Assume that all the functions therein are strictly differentiable at $(\bar{x}, \bar{y})$ and $\bar{x}$, respectively, and that the bilevel program is partially calm at $(\bar{x}, \bar{y})$. Assume further that $(\bar{x}, \bar{y})$ is lower-level regular, that $\bar{x}$ is upper-level regular, and that the solution map $S$ in (1.1) is $\mu$-inner semicontinuous at $(\bar{x}, \bar{y})$. Then there are numbers $\kappa>0, \lambda_{1}, \ldots, \lambda_{p}, \beta_{1}, \ldots, \beta_{p}$, and $\alpha_{1}, \ldots, \alpha_{k}$ such that

$$
\begin{gathered}
\nabla_{x} F(\bar{x}, \bar{y})+\sum_{i=1}^{p}\left(\beta_{i}-\kappa \lambda_{i}\right) \nabla_{x} g_{i}(\bar{x}, \bar{y})+\sum_{j=1}^{m} \alpha_{j} \nabla h_{j}(\bar{x})=0 \\
\nabla_{y} F(\bar{x}, \bar{y})+\kappa \nabla_{y} \varphi(\bar{x}, \bar{y})+\sum_{i=1}^{p} \beta_{i} \nabla_{y} g_{i}(\bar{x}, \bar{y})=0 \\
\nabla_{y} \varphi(\bar{x}, \bar{y})+\sum_{i=1}^{p} \lambda_{i} \nabla_{y} g_{i}(\bar{x}, \bar{y})=0
\end{gathered}
$$

with the following complementary slackness conditions:

$$
\begin{aligned}
& \lambda_{i} \geq 0, \quad \lambda_{i} g_{i}(\bar{x}, \bar{y})=0 \text { for all } i=1, \ldots, p, \\
& \beta_{i} \geq 0, \quad \beta_{i} g_{i}(\bar{x}, \bar{y})=0 \text { for all } i=1, \ldots, p, \\
& \alpha_{j} \geq 0, \quad \alpha_{j} h_{j}(\bar{x})=0 \text { for all } j=1, \ldots, m .
\end{aligned}
$$

Proof. Following the lines in the proof of [8, Theorem 3.1] under the assumptions made, we find numbers $\kappa>0, \beta_{1}, \ldots, \beta_{p}$, and $\alpha_{1}, \ldots, \alpha_{m}$ satisfying the sign and complementary slackness conditions (6.7) and (6.8) and such that the KKT condition

$$
\begin{aligned}
& 0 \in \nabla F(\bar{x}, \bar{y})+\kappa \nabla \varphi(\bar{x}, \bar{y})+(\kappa \partial(-\mu)(\bar{x}), 0) \\
&+\sum_{i=1}^{p} \beta_{i} \nabla g_{i}(\bar{x}, \bar{y})+\sum_{j=1}^{m} \alpha_{j}\left(\nabla h_{j}(\bar{x}), 0\right)
\end{aligned}
$$

holds for the single-level problem (4.5) under the imposed partial calmness at $(\bar{x}, \bar{y})$. Observe that

$$
\partial(-\mu)(\bar{x}) \subset-\bar{\partial} \mu(\bar{x})
$$

and that the lower-level regularity (6.1) reduce to the qualification condition (5.17) in this case. Then by using inner semicontinuous assumption on $S$, we apply Corollary 5.5 to get inclusion (5.20) for the convexified subdifferential of the marginal function. Incorporating this into (6.9) allows us to find multipliers $\lambda=\left(\lambda_{1}, \ldots, \lambda_{p}\right) \in \Lambda(\vec{x}, \bar{y})$ from the set (5.19) defined by only the inequality constraints such that conditions $(6.4),(6.5)$, and (6.6) are satisfied together with

$$
\begin{aligned}
& \nabla_{x} F(\bar{x}, \bar{y})+\kappa \nabla_{x} \varphi(\bar{x}, \bar{y})-\kappa\left[\nabla_{x} \varphi(\bar{x}, \bar{y})+\sum_{i=1}^{p} \lambda_{i} \nabla_{x} g_{i}(\bar{x}, \bar{y})\right] \\
& +\sum_{i=1}^{p} \beta_{i} \nabla_{x} g_{i}(\bar{x}, \bar{y})+\sum_{j=1}^{m} \alpha_{j} \nabla h_{j}(\bar{x})=0
\end{aligned}
$$

Collecting the like terms in the latter equation completes the proof of the theorem. 
Remark 6.2 (comparison with known necessary conditions for smooth bilevel programs). The result of Theorem 6.1 is different from that given in [8, Theorem 3.1] in finite dimensions under about the same assumptions (just the inner semicontinuity of $S(x)$ is required in [8] instead of its $\mu$-inner semicontinuity) due to the absence of convexification in (6.3) and thus involving much less unknown parameters in comparison with [8]. Note that the form of the necessary optimality conditions in Theorem 6.1 is similar to those given in [24, Theorem 4.1] under the convexity of the marginal function $\mu(x)$ and in [25, Theorem 4.1] on the concavity of the function $\varphi(x, y)-\mu(x)$ while with no inner semicontinuity of the solution map. The following example describes a situation when the assumptions in $[24,25]$ are not met while those in Theorem 6.1 are satisfied and allow us to find an optimal solution.

Example 6.3 (solving bilevel programs by means of Theorem 6.1). Consider the following bilevel program with smooth initial data:

$$
\text { minimize } F(x, y):=-y \text { subject to } y \in S(x),
$$

where $S: \mathbb{R} \rightrightarrows \mathbb{R}$ is the solution map for the lower-level problem:

$$
\begin{array}{ll}
\operatorname{minimize} & \varphi(x, y):=-y^{2}+x^{4}-3 x^{2}+1 \text { subject to } \\
& y \in G(x):=\left\{y \in \mathbb{R} \mid y+x^{2}-1 \leq 0 ;-y+x^{2}-1 \leq 0\right\} .
\end{array}
$$

It is easy to check that the bilevel program in this example admits an optimal solution with $x$ belonging to the interval $[-1,1]$. Furthermore, we have

$$
S(x)=\left\{-x^{2}+1, x^{2}-1\right\} \text { and } \mu(x)=-x^{2} \text { for } x \in[-1,1] .
$$

Thus neither the marginal function $\mu(x)$ is convex nor the difference function $\varphi(x, y)-\mu(x)=$ $-y^{2}+x^{4}-2 x^{2}+1$ is concave as it is required in $[24,25]$.

On the other hand, we see that the solution map $S$ is inner semicontinuous at any point $(x, y) \in \operatorname{gph} S$ and the lower-regularity assumption (6.1) is satisfied everywhere but $(-1,0$ and $(1,0)$; the upper-regularity is automatic due to the absence of inequality constraints on the upper level. Applying Theorem 6.1, we compute

$$
\begin{array}{ll}
\nabla F(x, y)=(0,-1), & \nabla \varphi(x, y)=\left(4 x^{3}-6 x,-2 y\right), \\
\nabla g_{1}(x, y)=(2 x, 1), & \nabla g_{2}(x, y)=(2 x,-1)
\end{array}
$$

and hence arrive at the relationships

$$
\begin{aligned}
& 0=\left(\beta_{1}-\kappa \lambda_{1}\right) 2 x+\left(\beta_{2}-\kappa \lambda_{2}\right) 2 x, \\
& 0=-1+\kappa(-2 y)+\beta_{1}(1)+\beta_{2}(-1), \\
& 0=-2 y+\lambda_{1}(1)+\lambda_{2}(-1) \\
& 0=\lambda_{1}\left(y+x^{2}-1\right)=\lambda_{2}\left(-y+x^{2}-1\right), \\
& 0=\beta_{1}\left(y+x^{2}-1\right)=\beta_{2}\left(-y+x^{2}-1\right)
\end{aligned}
$$

with $\kappa>0$ and all the nonnegative multipliers. Solving the above system gives us the points $(x, y) \in\{(0,1),(0,-1),(1,0),(-1,0)\}$ suspicious for optimality. Comparing the value of the upper-level objective at these point, we arrive at the pair $(\bar{x}, \bar{y})=(0,1)$ and check finally that the given bilevel program is partially calm at $(0,1)$. Thus we have found the optimal solution to the bilevel program under consideration by using Theorem 6.1.

The next result addresses the bilevel programs (4.1)-(4.3) with nonsmooth Lipschitzian data. In comparison with [8, Theorem 5.1] it contains much less unknown parameters in the necessary optimality conditions improving also a number of previous results in this direction. Note that the smooth case considered in Theorem 6.1 has certain specific features, which do not have any analogs in the nonsmooth settings. 
Theorem 6.4 (necessary optimality conditions for Lipschitzian bilevel programs, I). Let $(\bar{x}, \bar{y})$ be a local optimal solution to the bilevel program (4.1)-(4.3), where all the functions are locally Lipschitzian around $(\bar{x}, \bar{y})$ and $\bar{x}$, respectively. Assume that the program is partially calm at $(\bar{x}, \bar{y})$, that the lower constraint functions $g_{i}$ satisfy the $Q C(5.25)$ at $(\vec{x}, \bar{y})$, that $\bar{x}$ is upper-level regular (6.2), and that the solution map $S$ in (1.1) is $\mu$-inner semicontinuous at $(\bar{x}, \bar{y})$. Then there exist a number $\nu>0$, multipliers $\lambda_{1}, \ldots, \lambda_{p}, \beta_{1}, \ldots, \beta_{p}$, and $\alpha_{1}, \ldots, \alpha_{m}$ as well as an element $u^{*} \in X^{*}$ such that conditions (6.6)-(6.8) are satisfied together with

$$
\begin{aligned}
& \left(u^{*}, 0\right) \in \bar{\partial} \varphi(\bar{x}, \bar{y})+\sum_{i=1}^{p} \lambda_{i} \bar{\partial} g_{i}(\bar{x}, \bar{y}) \text { and } \\
& \left(u^{*}, 0\right) \in \partial \varphi(\bar{x}, \bar{y})+\nu \partial F(\bar{x}, \bar{y})+\sum_{i=1}^{p} \beta_{i} \partial g_{i}(\bar{x}, \bar{y})+\sum_{j=1}^{m} \alpha_{j}\left(\partial h_{j}(\bar{x}), 0\right) .
\end{aligned}
$$

Proof. Observe first that the qualification condition (5.25) implies the lower-level regularity (6.1) of $(\bar{x}, \bar{y})$. Following the proof of [8, Theorem 5.1] under the assumptions made, we find numbers $\kappa>0, \beta_{1}, \ldots, \beta_{p}, \alpha_{1}, \ldots, \alpha_{m}$ satisfying the sign and complementary slackness conditions (6.7) and (6.8) such that

$$
\begin{aligned}
& 0 \in \partial F(\bar{x}, \bar{y})+\kappa \partial \varphi(\bar{x}, \bar{y})+(\kappa \partial(-\mu)(\bar{x}), 0) \\
&+\sum_{i=1}^{p} \beta_{i} \partial g_{i}(\bar{x}, \bar{y})+\sum_{j=1}^{k} \alpha_{j}\left(\partial h_{j}(\bar{x}), 0\right)
\end{aligned}
$$

in terms the limiting subdifferential therein, where the marginal function $\mu(x)$ is Lipschitz continuous around $\bar{x}$. Taking into account that $\partial(-\mu)(\bar{x}) \subset-\bar{\partial} \mu(\bar{x})$ and choosing $u^{*} \in \bar{\partial} \mu(\bar{x})$, we have the inclusion

$$
\kappa\left(u^{*}, 0\right) \in \partial F(\bar{x}, \bar{y})+\kappa \partial \varphi(\bar{x}, \bar{y})+\sum_{i=1}^{p} \beta_{i} \partial g_{i}(\bar{x}, \bar{y})+\sum_{j=1}^{m} \alpha_{j}\left(\partial h_{j}(\bar{x}), 0\right) .
$$

Employing Theorem 5.9 gives us multipliers $\lambda_{i}$ satisfying the sign and complementary slackness conditions in (6.6) and such that the first inclusion in (6.10) holds. To get the second inclusion in (6.10), we divide (6.11) by $\kappa>0$ denoting $\nu:=\kappa^{-1}$ and keeping the same notation for the obtained multipliers $\beta_{i}$ and $\alpha_{j}$, which still satisfy the conditions in (6.7) and (6.8).

\section{Entering Hölder Subgradients}

The concluding section of the paper is devoted to developing some calculus results for the general class of the so-called Hölder subgradients (including Fréchet and proximal ones), which revolve around subdifferential estimates for marginal functions and then incorporate them into deriving new necessary optimality conditions for smooth and nonsmooth bilevel programs. For simplicity we consider here the bilevel programs

$$
\begin{aligned}
\operatorname{minimize} & F(x, y) \quad \text { subject to } \quad y \in S(x) \\
& \text { with } S(x)=\{y \in G(x) \mid \varphi(x, y)=\mu(x)\}, \\
& \mu(x)=\inf \{\varphi(x, y) \mid y \in G(x)\}, \\
& \text { and } G(x)=\left\{y \in Y \mid g_{i}(x, y) \leq 0, i=1, \ldots, p\right\}
\end{aligned}
$$

with no constraints on the upper level. Similar to the previous section, our approach involves subdifferential upper estimates for the marginal function (1.7) while subgradients differential from those in Section 6 and calculus rules for them, which are of their own interest. 
Recall that $u^{*} \in X^{*}$ is a Hölder subgradient of order $s \geq 0$ for a function $\varphi: X \rightarrow \bar{R}$ at $\bar{x} \in \operatorname{dom} \varphi$ if there are $C \geq 0$ and $r>0$ such that

$$
\left\langle u^{*}, x-\bar{x}\right\rangle \leq \varphi(x)-\varphi(\bar{x})+C\|x-\bar{x}\|^{1+s} \text { for all } x \in B_{r}(\bar{x}) .
$$

The collection of all $x^{*}$ satisfying (7.2) is called the s-Hölder subdifferential of $\varphi$ at $\bar{x}$ and is denoted by $\partial_{H(s)}^{+}(\bar{x})$. The case of $s=0$ in (7.2) is known as the Frceeher/regular subdifferential denoted by $\widehat{\partial} \varphi(\bar{x}) R$ while the case of $s=1$ corresponds to the so-called proximal subdifferential $\partial_{p} \varphi(\bar{x})$. Observe the inclusion

$$
\partial_{H\left(s_{1}\right)}^{+}(\bar{x}) \subset \partial_{H\left(s_{2}\right)}^{+}(\bar{x}) \text { whenever } s_{2} \leq s_{1} .
$$

We also consider the upper Hölder subdifferentials of $\varphi$ at $\bar{x} \in \operatorname{dom} \varphi$ defined by

$$
\partial_{H(s)}^{+} \varphi(\bar{x}):=-\partial_{H(s)}(-\varphi)(\bar{x})
$$

and use the simplify " $\diamond$ " notation for all the $s$-Hölder subdifferentials as $s \geq 0$ unless otherwise stated as in the case of the Fréchet one. Similarly to Section 2 we can define the $s$-Hölder normal cone and coderivative using the unified " $\diamond$ " notation for them. It is not hard to check that

$$
\partial_{\diamond} \varphi(\bar{x})=\left\{x^{*} \in X^{*} \mid\left(x^{*},-1\right) \in N_{\diamond}((\bar{x}, \varphi(\bar{x})) ; \operatorname{epi} \varphi)\right\} .
$$

The following theorem establishes new upper estimates for Hölder subgradients of the marginal function (1.7) in the form significantly different from those in Section 5. For the case of Fréchet subgradients, i.e., for $s=0$ in (7.2), it was obtained in [18, Theorem 1] with a different proof.

Theorem 7.1 (upper estimate for Hölder subgradients of marginal functions). Assume that $\partial_{\diamond}^{+} \varphi(\bar{x}, \bar{y}) \neq \emptyset$. Then we have the inclusion

$$
\partial_{\diamond} \mu(\bar{x}) \subset \bigcap_{\left(x^{*}, y^{*}\right) \in \partial_{\diamond}^{+} \varphi(\bar{x}, \bar{y})}\left[x^{*}+D_{\diamond}^{*} G(\bar{x}, \bar{y})\left(y^{*}\right)\right] .
$$

Proof. Take any Hölder subgradient $u^{*} \in \partial_{\diamond} \mu(\bar{x})$ and for a fixed number $s \geq 0$ in (7.2) find $C_{1} \geq 0$ and $r_{1}>0$ such that

$$
\left\langle u^{*}, x-\bar{x}\right\rangle \leq \mu(x)-\mu(\bar{x})+C_{1}\|x-\bar{x}\|^{1+s} \text { for all } x \in B_{r_{1}}(\bar{x}) .
$$

Since $\bar{y} \in S(\bar{x})$, we have the inequality

$$
\left\langle u^{*}, x-\bar{x}\right\rangle \leq \mu(x)-\varphi(\bar{x}, \bar{y})+C_{1}\|x-\bar{x}\|^{1+s} \text { whenever } x \in B_{r_{1}}(\bar{x}) .
$$

Now fix $\left(x^{*}, y^{*}\right) \in \partial_{\diamond}^{+} \varphi(\bar{x}, \bar{y})$ and find by the definition of Hölder upper subgradients such numbers $C_{2} \geq 0$ and $r_{2}>0$ that

$$
\varphi(x, y)-\varphi(\bar{x}, \bar{y}) \leq\left\langle x^{*}, x-\bar{x}\right\rangle+\left\langle y^{*}, y-\bar{y}\right\rangle+C_{2}(\|x-\bar{x}\|+\|y-\bar{y}\|)^{1+s}
$$

for all $(x, y)$ with $\|x-\bar{x}\|+\|y-\bar{y}\|<r_{2}$. Denoting

$$
C:=2 \max \left\{C_{1}, C_{2}\right\} \text { and } r:=\min \left\{r_{1}, r_{2}\right\},
$$

for any $y \in G(x)$ with $\|x-\bar{x}\|+\|y-\bar{x}\|<r$, we get $\mu(x) \leq \varphi(x, y)$ and hence

$$
\begin{aligned}
\left\langle u^{*}, x-\bar{x}\right\rangle & \leq \varphi(x, y)-\varphi(\bar{x}, \bar{y})+C_{1}\|x-\bar{x}\|^{1+s} \\
& \leq\left\langle x^{*}, x-\bar{x}\right\rangle+\left\langle y^{*}, y-\bar{y}\right\rangle+C_{1}\|x-\bar{x}\|^{1+s}+C_{2}(\|x-\bar{x}\|+\|y-\bar{y}\|)^{1+s} \\
& \leq\left\langle x^{*}, x-\bar{x}\right\rangle+\left\langle y^{*}, y-\bar{y}\right\rangle+C(\|x-\bar{x}\|+\|y-\bar{y}\|)^{1+s} .
\end{aligned}
$$


That implies the upper estimate

$$
\left\langle u^{*}-x^{*}, x-\bar{x}\right\rangle+\left\langle y^{*}, y-\bar{y}\right\rangle \leq C(\|x-\bar{x}\|+\|y-\bar{y}\|)^{1+s}
$$

for any $(x, y) \in \operatorname{gph} G$ with $\|x-\bar{x}\|+\|y-\bar{y}\|<r$. Thus $u^{*}-x^{*} \in D_{\diamond}^{*} G(\bar{x}, \bar{y})\left(y^{*}\right)$, which gives $u^{*} \in x^{*}+D_{\diamond}^{*} G(\bar{x}, \bar{y})\left(y^{*}\right)$ and completes the proof of the the theorem.

The next result follows from Theorem 7.1 and provides a useful difference rule for Hölder subgradients. For $s=0$ it was obtained in [17, Theorem 3.1] with a different proof.

Corollary 7.2 (difference rule for Hölder subgradients). Let $\varphi_{i}, \varphi_{2}: X \rightarrow \bar{R}$ be finite at $\bar{x}$. Assuming that $\partial_{\diamond} \varphi_{2}(\bar{x}) \neq \emptyset$ we have inclusion

$$
\partial_{\diamond}\left(\varphi_{1}-\varphi_{2}\right)(\bar{x}) \subset \bigcap_{x^{*} \in \partial_{\diamond} \varphi_{2}(\bar{x})}\left[\partial_{\diamond} \varphi_{1}(\bar{x})-x^{*}\right] .
$$

If implies in turns the optimality condition

$$
\partial_{\diamond} \varphi_{2}(\bar{x}) \subset \partial_{\diamond} \varphi_{1}(\bar{x})
$$

provided that $\bar{x}$ is a local minimizer for the difference function $\varphi_{1}-\varphi_{2}$.

Proof. Let $\varphi(x, y):=y-\varphi 2(x)$, and let $G(x):=\left[\varphi_{1}(x), \infty\right)$. Then we have the representations

$$
\mu(x)=\inf \{\varphi(x, y) \mid y \in G(x)\}=\varphi_{1}(x)-\varphi_{2}(x) \text { and } \partial_{\diamond}^{+} \varphi(\bar{x}, \bar{y})=\left(-\partial_{\diamond} \varphi_{2}(\bar{x}), 1\right) .
$$

Employing now Theorem 7.1 gives us

$$
\partial_{\diamond} \mu(\bar{x}) \subset \bigcap_{\left(x^{*}, y^{*}\right) \in\left(-\partial_{\diamond} \varphi_{2}(\bar{x}), 1\right)}\left[x^{*}+D_{\diamond}^{*} G(\bar{x}, \bar{y})\left(y^{*}\right)\right] .
$$

Since $\partial_{\diamond} \varphi_{1}(\bar{x})=D_{\diamond}^{*} G(\bar{x}, \bar{y})(1)$, we get

$$
\partial_{\diamond} \mu(\bar{x}) \subset \bigcap_{x^{*} \in \partial_{\diamond} \varphi_{2}(\bar{x})}\left[\partial_{\diamond} \varphi_{1}(\bar{x})-x^{*}\right]
$$

which verifies (7.3). Inclusion (7.4) follows immediately from (7.3) by using the Fermat rule

$$
0 \in \partial_{\diamond}\left(\varphi_{1}-\varphi_{2}\right)(\bar{x})
$$

and thus we complete the proof.

To derive next necessary optimality conditions for bilevel programs, we recall the following well-known representations of limiting normal to sets described by inequality constraints; see, e.g., $[14$, Theorems 3.8 and 3.86].

Lemma 7.3 (limiting normals to inequality constraints). Let $g_{i}$ as $i=1, \ldots, p$ be locally Lipschitzian around $\bar{x}$, let

$$
\Omega_{i}:=\left\{x \in X \mid g_{i}(x) \leq 0\right\}, \quad i=1, \ldots, p,
$$

and let the qualification condition

$$
\left[\sum_{i \in I(\bar{x})} \lambda_{i} x_{i}^{*}=0, x_{i}^{*} \in \partial g_{i}(\vec{x}), \lambda_{i} \geq 0\right] \Longrightarrow\left[\lambda_{i}=0 \text { for all } i \in I(\bar{x})\right]
$$

be satisfied with $I(\bar{x})=\left\{i=1, \ldots, p \mid g_{i}(\bar{x})=0\right\}$. Then for any $x^{*} \in N\left(\bar{x} ; \bigcap_{i=1}^{p} \Omega_{i}\right)$ we have

$$
x^{*} \in \sum_{i \in I(\bar{x})} \lambda_{i} \partial g_{i}(\bar{x}) \text { with some } \lambda_{i} \geq 0 .
$$


Now we are ready to establish the main result of this section, which provides necessary optimality conditions for bilevel programs with Lipschitzian data essentially different from those in Theorem 6.4 in both assumptions and conclusions. In particular, that we do not impose the $\mu$ inner semicontinuity of the solution map $S(x)$ in (7.1) while require the Frćhet subdifferentiability of the marginal function at the solution point.

Theorem 7.4 (necessary optimality condition for Lipschitzian bilevel programs, II). Let $(\bar{x}, \bar{y})$ be a local optimal solution to the bilevel program (7.1). Assume that all the functions involved are locally Lipschitzian around $(\bar{x}, \bar{y})$ and that the bilevel program in consideration is partially calm at $(\bar{x}, \bar{y})$. Suppose further that the qualification condition $(7.5)$ is satisfied and that $\widehat{\partial} \mu(\bar{x}) \neq \emptyset$. Then there are a number $\nu>0$, nonnegative multipliers $\lambda_{i}$ and $\beta_{i}$ satisfying the complementary slackness condition (6.6) and (6.7) as $i=1, \ldots, p$, as well as $u^{*} \in X^{*}$ such that

$$
\begin{aligned}
& \left(u^{*}, 0\right) \in \partial \varphi(\bar{x}, \bar{y})+\sum_{i=1}^{p} \lambda_{i} \partial g_{i}(\bar{x}, \bar{y}) \text { and } \\
& \left(u^{*}, 0\right) \in \partial \varphi(\bar{x}, \bar{y})+\nu \partial F(\bar{x}, \bar{y})+\sum_{i=1}^{p} \beta_{i} \partial g_{i}(\bar{x}, \bar{y}) .
\end{aligned}
$$

Proof. It follows from Proposition 4.1 and the constraint penalization via the indicator function $\delta(\cdot ; \mathrm{gph} G)$ that $(\bar{x}, \bar{y})$ a local optimal solution to the unconstrained problem

$$
\text { minimize } F(x, y)+\kappa(\varphi(x, y)-\mu(x))+\delta((x, y) ; \operatorname{gph} G)
$$

Applying the necessary optimality condition (7.4) from Proposition 7.2 with $s=0$ therein to the difference function in (7.7), we get

$$
(\kappa \widehat{\partial} \mu(\bar{x}), 0) \subset \widehat{\partial}(F(\cdot)+\kappa \varphi(\cdot)+\delta(\cdot ; \operatorname{gph} G))(\bar{x}, \bar{y}) .
$$

On the other hand, it follows from the proof of [14, Theorem 1.108] that

$$
(\widehat{\partial} \mu(\bar{x}), 0) \subset \widehat{\partial}(\varphi(\cdot)+\delta(\cdot ; \operatorname{gph} G))(\bar{x}, \bar{y}) .
$$

Passing to the larger limiting subdifferentials on the right-hand sides of (7.8) and (7.9) and employing then the subdifferential sum rule from [14, Theorem 2.33] with taking into account the Lipschitz continuity of $\varphi$ and $F$, we have

$$
\begin{aligned}
& (\kappa \widehat{\partial} \mu(\bar{x}), 0) \subset \partial F(\bar{x}, \bar{y})+\kappa \partial \varphi(\bar{x}, \bar{y})+N((\bar{x}, \bar{y}) ; \operatorname{gph} G), \\
& (\widehat{\partial} \mu(\bar{x}), 0) \subset \partial \varphi(\bar{x}, \bar{y})+N((\bar{x}, \bar{y}) ; \operatorname{gph} G) .
\end{aligned}
$$

It follows from 7.3 by the assumed qualification condition (7.5) that

$$
N((\vec{x}, \bar{y}) ; \operatorname{gph} G) \subset \bigcup\left\{\sum_{i=1}^{p} \lambda_{i} \partial g_{i}(\bar{x}, \bar{y}) \mid \lambda_{i} \geq 0, \lambda_{i} g_{i}(\bar{x}, \bar{y})=0 \text { as } i=1, \ldots, p\right\} .
$$

That implies the existence of multipliers $\lambda_{i}$ and $\beta_{i}$ satisfying the sign and complementarity slackness conditions in (6.6) and (6.7) as well as an element $u^{*} \in X^{*}$ such that

$$
\begin{aligned}
& \left(u^{*}, 0\right) \in \partial \varphi(\bar{x}, \bar{y})+\sum_{i=1}^{p} \lambda_{i} \partial g_{i}(\bar{x}, \bar{y}) \text { and } \\
& \kappa\left(u^{*}, 0\right) \in \partial F(\bar{x}, \bar{y})+\kappa \partial \varphi(\bar{x}, \bar{y})+\sum_{i=1}^{p} \beta_{i} \partial g_{i}(\bar{x}, \bar{y}) .
\end{aligned}
$$


Dividing the latter inclusion by $\kappa>0$ and denoting $\nu:=k^{-1}$ while keeping the same notation for the modified multipliers $\beta_{i}$, we arrive at the necessary optimality conditions (7.6).

The following specification of the above theorem holds for bilevel programs with initial data strictly differentiable at the reference optimal solution.

Corollary 7.5 (necessary optimality conditions for smooth bilevel programs, II). Let $(\bar{x}, \bar{y})$ be a local optimal solution to the bilevel program (7.1), where the functions $\varphi, F$, and $g_{i}$ are strictly differentiable at the point $(\bar{x}, \bar{y})$ that is lower-level regular as in (6.1). Assume also that the bilevel program is partially calm at $(\bar{x}, \bar{y})$ and that $\widehat{\partial} \mu(\bar{x}) \neq \emptyset$. Then there are multipliers $\nu_{i}$ and $\beta_{i}$ as $i=1, \ldots, p$ such that $\beta_{i}$ satisfy the sign a complementarity slackness condition (6.7), $\nu_{i}$ satisfy the complementarity slackness condition

$$
\nu_{i} g_{i}(\bar{x}, \bar{y})=0 \text { for all } i=1, \ldots, p,
$$

and the following equality hold:

$$
\begin{aligned}
& \nabla F(\bar{x}, \bar{y})+\sum_{i=1}^{p} \alpha_{i} \nabla g_{i}(\bar{x}, \bar{y})=0 \\
& \nabla_{y} \varphi(\bar{x}, \bar{y})+\sum_{i=1}^{p} \beta_{i} \nabla_{y} g_{i}(\bar{x}, \bar{y})=0
\end{aligned}
$$

Proof. It follows from Theorem 7.4 by taking into account that

$$
\partial f(\bar{x}, \bar{y})=\left\{\nabla_{x} f(\bar{x}, \bar{y}), \nabla_{y} f(\bar{x}, \bar{y})\right\}
$$

for any function $f: X \rightarrow \mathbb{R}$ strictly differentiable at $(\bar{x}, \bar{y})$ and then by collecting the like terms in the corresponding relationships (7.6).

Observe that Corollary 7.5 does not ensure the nonnegativity of the multipliers $\nu_{i}$. Note also that a slight modification of the proof of Theorem 7.4 allows us to obtain the necessary optimality conditions in Corollary 7.5 under merely Fréchet differentiability of the functions $\varphi$ and $F$ at $(\bar{x}, \bar{y})$.

Remark 7.6 (necessary optimality conditions for bilevel programs with convex marginal functions). If we assume that the marginal function $\mu(x)$ in (7.1) is convex around $\bar{x}$, then the challenging assumption $\widehat{\partial} \mu(\bar{x}) \neq \emptyset$ is automatic. Thus in this case the results of Theorem 7.5 and Corollary 7.5 can be unconditionally applied under the conventional assumptions made; cf. $[24,25,27]$ and the references therein.

We conclude this section with an example illustrating the application of the obtained necessary optimality conditions in the case when no known result can be applied.

Example 7.7 (smooth bilevel programs with nonconvex while Fréchet differentiable marginal functions). Consider a smooth bilevel program given by

$$
\text { minimize } F(x, y):=-y \text { subject to } y \in S(x),
$$

where $S: \mathbb{R} \rightrightarrows \mathbb{R}$ is the solution map for the lower-level problem:

$$
\begin{gathered}
\text { minimize } \varphi(x, y):=-y^{2} \quad \text { subject to } \\
y \in G(x):=\left\{y \in \mathbb{R} \mid-x+y^{4}-1 \leq 0, x+y^{4}-1 \leq 0\right\} .
\end{gathered}
$$

It is easy to see that this program admits an optimal solution. Then we calculate the lower-level solution map by $S(x)=\{ \pm \sqrt{1-|x|}\}$ and the marginal function by $\mu(x)=-\sqrt{1-|x|}$. In this 
case neither $\mu(x)$ is convex nor the difference function $\varphi(x, y)-\mu(x)=-y^{2}+\sqrt{1-|x|}$ is concave, and hence the related results of [24, Theorem 4.2] and [25, Theorem 4.1] are not applicable.

On the other hand, the marginal function is everywhere Fréchet subdifferentiable, and we can apply the necessary optimality conditions of Corollary 7.5. They give the equations:

$$
\begin{aligned}
& \nu_{1}(-1)+\nu_{2}(1)=0, \\
& -1+\nu_{1}\left(4 y^{3}\right)+\nu_{2}\left(4 y^{3}\right)=0, \\
& -2 y+\beta_{1}\left(4 y^{3}\right)+\beta_{2}\left(4 y^{3}\right)=0, \\
& \nu_{1}\left(-x+y^{4}-1\right)=\nu_{2}\left(x+y^{4}-1\right)=0, \\
& \beta_{1}\left(-x+y^{4}-1\right)=\beta_{1}\left(x+y^{4}-1\right)=0, \beta_{1} \geq 0, \beta_{2} \geq 0 .
\end{aligned}
$$

Solving this system, we obtain the points $(x, y)=(0, \pm 1)$. Comparing the upper-level objective selects the point $(0,1)$. Finally, we check that the bilevel program under consideration is partially calm at $(0,1)$, and thus it is the optimal solution

\section{References}

[1] T. Q. Bao, P. Gupta, B. S. Mordukhovich (2007), Necessary conditions in multiobjective optimization with equilibrium constraints, JOTA 135, 179-203.

[2] A. V. Borovik, A. Borovik (2010), Mirrors and Reflections: The Geometry of Finite Reflection, Springer, New York.

[3] J. M. Borwern, Q. J. ZhU (2005), Techniques of Variational Analysis, Springer, New York.

[4] J. V. Burke, S. Deng (2002), Weak sharp minima revisited, Part I: Basic theory, Control and Cybernetics 31, 439-469.

[5] F. H. Clarke (1983), Optimization and Nonsmooth Analysis, Wiley, New York.

[6] S. Dempe (2003), Foundations of Bilevel Programming, Kluwer, Dordrecht, The Netherlands.

[7] S. Dempe, J. DutTa (2011), Is bilevel programming a special case of mathematical programming with complementarity constraints? Math. Program., to appear.

[8] S. Dempe, J. DutTA, B. S. Mordukhovich (2007), New necessary optimality conditions in optimistic bilevel programming, Optimization 56, 577-604.

[9] S. Dempe, B. S. Mordukhovich, A. B. Zemhoko (2011), Sensitivity analysis for two-level value functions with applications to bilevel programs, preprint.

[10] S. Dempe, A. B. ZEMKoho (2011), The bilevel programming problem: Reformulations, constraint qualifications and optimality conditions, Math. Program., to appear.

[11] N. Dinh, B. S. Mordukhovich, T. T. A. NGHiA (2010), Subdifferentials of value functions and optimality conditions for DC and bilevel infinite and semi-infinite programs, Math. Program. 123, 101-138.

[12] J. DutTA, S. DEMPE (2006), Bilevel programming with convex lower level problems. In: Optimization with Multivalued Mappings (S. Dempe and V. Kalashnikov, eds.), Springer, New York.

[13] R. Henrion, T. M. Surowiec (2011), On calmness conditions in convex bilevel programming, Applic. Analysis, to appear. 
[14] B. S. MordukHovich (2006), Variational Analysis and Generalized Differentiation, I: Basic Theory, Springer, Berlin.

[15] B. S. MoRdukHovich (2006), Variational Analysis and Generalized Differentiation, II: Applications, Springer, Berlin.

[16] B. S. MoRdukHovich, N. M. NAM (2005), Variational stability and marginal functions via generalized differentiation, Math. Oper. Res. 30, 800-816.

[17] B. S. Mordukhovich, N. M. NAM, N. D. YEN (2006), Fréchet subdifferential calculus and optimality conditions in nondifferentiable programming, Optimization 55, 685-708.

[18] B. S. MordukHovich, N. M. NAM, N. D. YEN (2009), Subgradients of marginal functions in parametric mathematical programming. Math. Program. 116, 369-396.

[19] J. V. OUTRATA (1990), On the numerical solution of a class of Stackelberg problems, ZORMethods and Models of Oper. Res. 34, 255-277.

[20] R. T. RockAfELLAR (1979), Directionally Lipschitzian functions and subdifferential calculus, Proc. London Math. Soc. 39, 331-355.

[21] R. T. Rockafellar (1985), Extensions of subgradient calculus with applications to optimization, Nonlinear Anal. 9, 665-698.

[22] R. T. Rockafellar, R-J. Wets (1998), Variational Analysis, Springer, Berlin.

[23] W. Schirotzek (2007), Nonsmooth Analysis, Springer, Berlin.

[24] J. J. YE (2004), Nondifferentiable multiplier rules for optimization and bilevel optimization problems, SIAM J. Optim. 15, 252-274.

[25] J. J. YE (2006), Constraint qualifications and KKT conditions for bilevel programming problems, Math. Oper. Res. 31, 811-824.

[26] J. J. YE (2011), Necessary optimality conditions for multiobjective bilevel programs, Math. Oper. Res., to appear.

[27] J. J. YE, D. L. ZHU (1995), Optimality conditions for bilevel programming problems, Optimization 33, 9-27.

[28] J. J. YE, D. L. ZHU (2010), New necessary optimality conditions for bilevel programs by combining MPEC and the value function approach, SIAM J. Optim. 20, 1885-1905.

[29] Y. Zhou, B. S. MoRdukhovich, N. XIU (2011), Complete characterizations of local weak sharp minima with applications to semi-infinite programming and semi-infinite complementarity, Nonlinear Anal., to appear. 